https://helda.helsinki.fi

\title{
BRST Quantization of Unimodular Gravity
}

\section{Upadhyay, Sudhaker}

2017-06

Upadhyay , S , Oksanen , M \& Bufalo , R 2017 , ' BRST Quantization of Unimodular Gravity '

, Brazilian Journal of Physics, vol. 47 , no. 3 , pp. 350-365 . https://doi.org/10.1007/s13538-017-0500-5

http://hdl.handle.net/10138/228030

https://doi.org/10.1007/s13538-017-0500-5

unspecified

acceptedVersion

Downloaded from Helda, University of Helsinki institutional repository.

This is an electronic reprint of the original article.

This reprint may differ from the original in pagination and typographic detail.

Please cite the original version. 


\title{
BRST Quantization of Unimodular Gravity
}

\author{
Sudhaker Upadhyay ${ }^{d 1}$, Markku Oksanen ${ }^{2}$ and Rodrigo Bufalo ${ }^{43}$ \\ ${ }^{a}$ Centre for Theoretical Studies, Indian Institute of Technology Kharagpur \\ Kharagpur-721302, India \\ ${ }^{b}$ Department of Physics, University of Helsinki, P.O. Box 64 \\ FI-00014 Helsinki, Finland \\ ${ }^{c}$ Instituto de Física Teórica, Universidade Estadual Paulista \\ Rua Dr. Bento Teobaldo Ferraz 271, Bloco II, 01140-070 São Paulo, SP, Brazil
}

\begin{abstract}
We study the quantization of two versions of unimodular gravity, namely, fully diffeomorphisminvariant unimodular gravity and unimodular gravity with fixed metric determinant utilizing standard path integral approach. We derive the BRST symmetry of effective actions corresponding to several relevant gauge conditions. We observe that for some gauge conditions, the restricted gauge structure may complicate the formulation and effective actions, in particular, if the chosen gauge conditions involve the canonical momentum conjugate to the induced metric on the spatial hypersurface. The BRST symmetry is extended further to the finite field-dependent BRST transformation, in order to establish the mapping between different gauge conditions in each of the two versions of unimodular gravity.
\end{abstract}

PACS: 04.50.Kd, 04.60.-m, 11.15.-q, 11.30.-j

\section{Introduction}

Motivated by different purposes and scenarios a considerable attention has been paid to alternative gravitational theories in recent years. In particular, substantial efforts have been invested in understanding the so-called cosmological constant problem [1 3 , more precisely why the vacuum energy does not produce a huge value for the cosmological constant, many orders of magnitude above the observed value. Within this context a gravitational theory, nearly as old as general relativity (GR) itself [4], the so-called unimodular gravity (UG) [5], has once again been analyzed [6] as a potential way to approach the problem.

Originally, the idea of unimodular gravity was conceived when Einstein considered the unimodular condition $[4], \sqrt{-g}=1$, as a convenient way to partially fix a coordinate system in GR. The definition of unimodular gravity is usually based on the invariance under a restricted group of diffeomorphisms that leave the determinant of the metric invariant, so that the determinant of the metric can be set equal to a fixed scalar density $\epsilon_{0}, \sqrt{-g}=\epsilon_{0}$. Alternatively, one could consider restricted diffeomorphisms that preserve the volume of spacetime [7]. The field equation for the metric is either the traceless Einstein equation or, due to the Bianchi identity, the Einstein equation with a cosmological constant 8].

In comparison with GR, making the cosmological constant an arbitrary constant of integration can be regarded as the key feature of unimodular gravity. In order to achieve it, however, there is

\footnotetext{
${ }^{1}$ E-mail: sudhakerupadhyay@gmail.com

${ }^{2}$ E-mail: markku.oksanen@helsinki.fi

${ }^{3}$ E-mail: rbufalo@ift.unesp.br
} 
no need to constrain the determinant of the metric. One can, therefore, either extend the above unimodular condition in order to enlarge its group of symmetry, e.g. by setting $\sqrt{-g}$ equal to the divergence of a vector density field via parameterization of the spacetime coordinates [9]. This kind of construction encompass the set of theories known as fully diffeomorphism-invariant extensions of unimodular gravity. The most prominent theories of this kind are the Henneaux-Teitelboim theory [10 and the fully diffeomorphism-invariant theory of unimodular gravity [11. The latter is no longer unimodular in the sense that there is no condition on the determinant of the metric. Nonetheless, it has been established how the theory is canonically related to the conventional unimodular theory of gravity [1].

Returning to the aforementioned cosmological constant problem, a highly speculative but interesting (formal) attempt to address this problem in unimodular gravity has been made in 6, 12 and carefully revised in [11], but with no decisive conclusion. Unimodular gravity has also been used in investigating other fundamental problems in gravitational theory. In particular, one may argue that since the bulk part of the Hamiltonian of unimodular gravity is nonvanishing, and the four-volume provides a cosmological time, unimodular gravity could offer a new perspective on the problem of time in quantum gravity and cosmology $8,13,14$. However, later it was shown that the problem of time persists in quantum unimodular gravity $[9]$.

In classical level it is well known that unimodular gravity produces the same physics as GR with a cosmological constant [8]. However, a natural concern arises when such equivalence is investigated in the quantum level, since a systematic and detailed study is necessary and any conclusion beyond formal realm is always very subtle within gravity. In addition, one may realize that quantization of each version of unimodular gravity can be regarded as a potential quantization of GR. Therefore, in order to shed a new light into several issues, analyses considering the canonical structure and path integral quantization 11 and radiative calculations [15] of unimodular gravity have been presented recently. Although very interesting and important conclusions were drawn from such studies, several formal aspects still need to be answered via deeper analysis within this scope. Hence, the implementation of BRST formulations of the unimodular gravity theories plays an interesting and important role in understanding the structure of these theories. The BRST formulation is known to be a powerful method for quantization of gauge theories, since it simplifies the proofs of renormalizability, unitarity and anomaly cancellation.

A suitable approach for such analysis consist in an extension of BRST symmetry realized by allowing the parameter to be finite and field-dependent, the so-called finite field-dependent BRST (FFBRST) symmetry [16. Ref. [16] deals with the issue of generalizing BRST symmetry in YangMills theories from the infinitesimal case to the finite case, while attempting to include the case of BRST-antiBRST symmetry by using the same approach as in the case of BRST symmetry, i.e., one that explicitly utilizes only a linear dependence on the corresponding Grassmann-odd parameters. The FFBRST symmetry transformations have found several applications in a wide area of theoretical high energy physics.

Within the most relevant results obtained from an analysis following FFBRST symmetry we may cite, for instance, a correct prescription for poles in the gauge field propagators in noncovariant gauges has been derived with the help of FFBRST transformation by connecting it to covariant gauges [17]. The long outstanding problem of divergent energy integrals in Coulomb gauge has also been regularized with the help of FFBRST transformation [18. The generalization of both on-shell and off-shell BRST as well as anti-BRST symmetries for Yang-Mills theory are demonstrated explicitly where these are shown to establish the mapping between various gauges of the theory [19]. The celebrated Gribov 
issue 2022 has also been addressed by connecting the Yang-Mills theory (possessing Gribov copies) to Gribov-Zwanziger theory (free from Gribov copies within a Gribov horizon) within the framework of FFBRST formulation (see refs. therein 23]). The FFBRST transformations have been applied successfully in the study of many other gauge theories 2432 .

An extension of FFBRST formulation has been established for various theories at quantum level [33, 34 utilizing Batalin-Vilkovisky (BV) formalism [35]. Lavrov and Lechtenfeld [36] suggests an alternative, w.r.t. [16], approach to generalize the BRST transformations in Yang-Mills theories, also by using a linear dependence on the corresponding Grassmann-odd parameter, naturally without having recourse to any quadratic dependence, since Ref. 36 does not deal with the case of BRSTantiBRST symmetry, and so any non-trivial quadratic dependence on the transformation parameters cannot occur. Moshin and Reshetnyak in Ref. 37 have systematically incorporated the case of BRST-antiBRST symmetry in Yang-Mills theories within the context of finite transformations that deals with the case of a quadratic dependence on the corresponding parameters. This follows from the calculation of the corresponding Jacobian and from investigating the resulting quantum action. The concept of finite BRST-antiBRST symmetry is further extended to the case of general gauge theories [38, 39] as well as supersymmetric (SUSY) theories [40], whereas Ref. [41, 42] generalizes the corresponding parameters to the case of arbitrary Grassmann-odd field-dependent parameters. We feel that the generalization of the BRST formalism could be useful in understanding the quantization of unimodular gravity theories.

The aim of the present paper is to investigate the features of the two unimodular gravity theories in the BRST as well as in generalized BRST framework. Specifically, we discuss several potential gauge conditions for the two unimodular gravity theories, one theory with full diffeomorphism-invariance and the other with fixed metric determinant. We compute the induced ghost action for each set of gauge conditions, and write down the path integral for each effective action. We demonstrate the nilpotent BRST symmetry of the effective action and the corresponding transition amplitude. Moreover, we extend the BRST symmetry by making the transformation parameter finite and field dependent in the case of unimodular gravity. The action is invariant under such a non-linear transformation of the fields. However, the functional measure is not covariant under the FFBRST transformations. We compute the non-trivial Jacobian for the functional measure under FFBRST transformation for the two cases of unimodular gravity in general gauge conditions. To illustrate this result we consider several gauge conditions in both the fully diffeomorphism-invariant theory and the theory with fixed metric determinant. Remarkably, we show that the FFBRST transformation with certain parameters connects different gauges of the given theories. In this way we are able to approach the different sets of gauge conditions. Suppose any calculation in one set of gauge conditions is unambiguous, a similar procedure for a different set of gauge conditions could possibly be arrived at if one were to establish a connection between the different sets of gauge conditions.

The paper is organized as follows. In section 2, we discuss a unimodular gravity theory extension endowed of fully diffeomorphism-invariant theory in various gauge conditions. The respective BRST symmetry transformations are derived and the gauge fixing and ghost action is determined as well. A similar analysis for unimodular gravity theory with a fixed metric determinant is presented subsequently in section 3. We analyse such theory in rather different gauges than the full diffeomorphism invariant case. Further, in section 4, we provide a review of the methodology for the FFBRST symmetry analysis in the case of fully diffeomorphism-invariant unimodular gravity. We compute the explicit expression for Jacobian under FFBRST transformation which depends on infinitesimal fielddependent parameter explicitly. Under these circumstances, we show that the Jacobian is responsible 
for the gauge connection between different transition amplitudes. To be specific, we connect harmonic gauge, synchronous gauge, axial gauge, Lorentz gauge and planar gauge to each other for the fully diffeomorphism-invariant case. Nonetheless, the unimodular Faddeev-Popov gauge, averaged metric determinant gauge and averaged metric trace gauge are connected to each other in the fixed metric determinant case. In the section 5 we summarize the results.

\section{Unimodular gravity with full diffeomorphism invariance}

We start our analysis with a brief review on the fully diffeomorphism-invariant unimodular gravity. But first, it shows to be convenient to revise the Henneaux-Teitelboim (HT) action [10]

$$
S_{\mathrm{HT}}=\int_{\mathcal{M}} d^{4} x\left(\frac{\sqrt{-g} R}{\kappa}-\lambda\left(\sqrt{-g}-\partial_{\mu} \tau^{\mu}\right)\right)+\oint_{\partial \mathcal{M}} d^{3} x\left(\frac{2}{\kappa} \sqrt{|\gamma|} \mathcal{K}-\lambda r_{\mu} \tau^{\mu}\right),
$$

where $\tau^{\mu}$ is a vector density, the gravitational coupling constant is denoted as $\kappa=16 \pi G$, $\gamma$ is the determinant of the induced metric on the boundary $\partial \mathcal{M}$ of spacetime, $\mathcal{K}$ is the extrinsic scalar curvature of $\partial \mathcal{M}$, and $r_{\mu}$ is the outward-pointing unit normal to the boundary $\partial \mathcal{M}$. The (fully diffeomorphisminvariant) unimodular condition has been introduced into the action (2.1) as a constraint multiplied by a Lagrange multiplier $\lambda$. The boundary term is included as in GR, so that the variational principle for the action is well defined without imposing boundary conditions on the derivatives of the metric.

The field equations consist of the Einstein equation, the equation for the cosmological constant variable,

$$
\nabla_{\mu} \lambda=0
$$

a (fully diffeomorphism-invariant) unimodular condition,

$$
\sqrt{-g}=\partial_{\mu} \tau^{\mu}
$$

The HT action (2.1) can indeed be derived from the UG action, Eq. (3.1), via parameterization of the spacetime coordinates 9 .

We consider now an alternative action that is also fully diffeomorphism-invariant and retains the classical equivalence with the other unimodular theories. The action is written as

$$
S_{\mathrm{DUG}}\left[g_{\mu \nu}, \lambda, V^{\mu}\right]=\int_{\mathcal{M}} d^{4} x \sqrt{-g}\left(\frac{R}{\kappa}-\lambda-V^{\mu} \nabla_{\mu} \lambda\right)+\frac{2}{\kappa} \oint_{\partial \mathcal{M}} d^{3} x \sqrt{|\gamma|} \mathcal{K},
$$

where the variable $V^{\mu}$ is a vector field. We shall refer to this theory as the fully diffeomorphisminvariant unimodular gravity (DUG). The action (2.4) is arguably the most transparent definition of such a theory. The action (2.4) consists of the Einstein-Hilbert action with a variable cosmological constant $\lambda$, and a constraint term for $\lambda$. The vector field $V^{\mu}$ acts as a Lagrange multiplier that ensures $\nabla_{\mu} \lambda$ is zero in every direction, and thus $\lambda$ is a constant. Classical solutions to the field equations defined by the action (2.4) are the same as for GR with a cosmological constant.

The Hamiltonian analysis follows straightforwardly for the DUG action when written in the Arnowitt-Deser-Misner (ADM) form [11]. After a systematic canonical procedure at an arbitrary gauge-fixing $\chi^{\mu}$, the path integral for the given theory is found to be [11]

$$
Z_{\mathrm{DUG}}=\mathcal{N}^{-1} \int \prod_{x} \mathcal{D} g_{\mu \nu} g^{00}(-g)^{-\frac{3}{2}} N \delta\left(\chi^{\mu}\right)\left|\operatorname{det}\left\{\chi^{\mu}, \mathcal{H}_{\nu}\right\}\right| \exp \left(\frac{i}{\hbar} S_{\mathrm{EH}}\left[g_{\mu \nu}, \Lambda\right]\right)
$$


where we denoted the super-Hamiltonian and super-momentum constraints collectively as $\mathcal{H}_{\nu}=$ $\left(\mathcal{H}_{T}, \mathcal{H}_{i}\right)$ and $S_{\mathrm{EH}}$ is the Einstein-Hilbert action with a cosmological constant

$$
S_{\mathrm{EH}}\left[g_{\mu \nu}, \Lambda\right]=\frac{1}{\kappa} \int_{\mathcal{M}} d^{4} x \sqrt{-g}(R-2 \Lambda)+\frac{2}{\kappa} \oint_{\partial \mathcal{M}} d^{3} x \sqrt{|\gamma|} \mathcal{K}
$$

It should be noted that the value of $\Lambda$ is not set by the action. The cosmological constant $\Lambda$ is an unspecified value of the variable $\lambda$.

The present theory has the advantage of enabling the use of the same (covariant) gauges for the diffeomorphism symmetry as in GR. In view of this, and bearing in mind the BRST analysis, let us recall that the infinitesimal (diffeomorphism) gauge transformation of the metric is written as

$$
\delta_{\xi} g_{\mu \nu}=\partial_{\rho} g_{\mu \nu} \xi^{\rho}+g_{\mu \rho} \partial_{\nu} \xi^{\rho}+g_{\rho \nu} \partial_{\mu} \xi^{\rho} .
$$

The inverse metric density is defined as

$$
\hat{g}^{\mu \nu}=\sqrt{-g} g^{\mu \nu},
$$

and its transformation under (2.7) is obtained as

$$
\delta_{\xi} \hat{g}^{\mu \nu}=\partial_{\rho}\left(\hat{g}^{\mu \nu} \xi^{\rho}\right)-\hat{g}^{\mu \rho} \partial_{\rho} \xi^{\nu}-\hat{g}^{\rho \nu} \partial_{\rho} \xi^{\mu} .
$$

\section{$2.1 \quad$ BRST Symmetry}

The BRST transformation for the full set of fields, metric field $g_{\mu \nu}$, Faddeev-Popov ghost fields $c^{\mu}, \bar{c}_{\nu}$, and Nakanishi-Lautrup auxiliary field $\eta_{\mu}$, can be obtained from the properties of infinitesimal diffeomorphisms as

$$
\begin{aligned}
\delta_{b} g_{\mu \nu} & =\left(\partial_{\rho} g_{\mu \nu} c^{\rho}+g_{\mu \rho} \partial_{\nu} c^{\rho}+g_{\rho \nu} \partial_{\mu} c^{\rho}\right) \theta \\
\delta_{b} c^{\mu} & =-c^{\nu} \partial_{\nu} c^{\mu} \theta \\
\delta_{b} \bar{c}_{\mu} & =\eta_{\mu} \theta \\
\delta_{b} \eta_{\mu} & =0
\end{aligned}
$$

The inverse metric density (2.8) transforms under (2.10a) as

$$
\delta_{b} \hat{g}^{\mu \nu}=\left(\partial_{\rho}\left(\hat{g}^{\mu \nu} c^{\rho}\right)-\hat{g}^{\mu \rho} \partial_{\rho} c^{\nu}-\hat{g}^{\rho \nu} \partial_{\rho} c^{\mu}\right) \theta .
$$

The BRST transformation of the metric is obtained from the infinitesimal diffeomorphism (2.7), with the replacement $\xi^{\rho} \rightarrow c^{\rho} \theta$. The transformation of the ghost $c^{\mu}$ was obtained from the commutator of vector fields generating the infinitesimal diffeomorphisms by replacing the vector components with an anticommuting field: $\left(c=c^{\mu} \partial_{\mu}\right)$

$$
-\frac{1}{2}[c, c]^{\mu}=-\frac{1}{2}\left(c^{\nu} \partial_{\nu} c^{\mu}-\partial_{\nu} c^{\mu} c^{\nu}\right)=-c^{\nu} \partial_{\nu} c^{\mu} .
$$

The transformation of the anti-ghost $\bar{c}_{\mu}$ is proportional to the auxiliary field $\eta_{\mu}$ that acts as a Lagrange multiplier of gauge conditions. The transformations (2.10a)-(2.10d) commute with spacetime differentiation. 


\subsection{Gauge fixing and ghost action}

Next we derive the BRST invariant gauge fixing and ghost action $S_{g f+g h}^{G}$ for different sets of gauge conditions, determining thus the respective path integral expression. Moreover, as aforementioned, we shall restrict our discussion to covariant and one non-covariant gauges for the DUG theory, while for the UG theory we will consider only non-covariant gauges.

\subsubsection{Harmonic gauge}

Let us start our analysis by choosing the transverse harmonic gauge,

$$
\partial_{\nu} \hat{g}^{\mu \nu}=0 .
$$

The gauge and ghost action can be written in the form

$$
S_{g f+g h}^{H}=\int d^{4} x\left(-\eta_{\mu} \partial_{\nu} \hat{g}^{\mu \nu}+\partial_{\nu} \bar{c}_{\mu}\left(\partial_{\rho}\left(\hat{g}^{\mu \nu} c^{\rho}\right)-\hat{g}^{\mu \rho} \partial_{\rho} c^{\nu}-\hat{g}^{\rho \nu} \partial_{\rho} c^{\mu}\right)\right) .
$$

In the action (2.14), the terms that involve the gauge conditions (2.13) could be absorbed into the gauge-fixing terms via a shift transformation of the auxiliary fields $\eta_{\mu}$. Still we choose to keep those terms in order to maintain manifest BRST invariance. Thus, we find the path integral in the harmonic gauge

$$
Z_{\mathrm{DUG}}^{H}=\mathcal{N}^{-1} \int \prod_{x} \mathcal{D} g_{\mu \nu} \mathcal{D} \eta_{\mu} \mathcal{D} \bar{c}_{\mu} \mathcal{D} c^{\nu} g^{00}(-g)^{-\frac{3}{2}} \exp \left(\frac{i}{\hbar}\left[S_{E H}\left[g_{\mu \nu}, \Lambda\right]+S_{g f+g h}^{H}\right]\right) .
$$

\subsubsection{Lorentz covariant $\alpha$-gauge}

A direct generalization of the above condition is the Lorentz covariant $\alpha$-gauge

$$
\partial_{\nu} \hat{g}^{\mu \nu}+\alpha \hat{g}_{\mathrm{R}}^{\mu \nu} \eta_{\nu}=0
$$

where $\hat{g}_{R}^{\mu \nu}$ is a fixed reference background metric density. The limit $\alpha \rightarrow 0$ reproduces the harmonic gauge. The gauge and ghost action with an arbitrary constant parameter $\alpha$ is written as

$$
S_{g f+g h}^{\alpha}=\int d^{4} x\left(-\frac{\alpha}{2} \hat{g}_{\mathrm{R}}^{\mu \nu} \eta_{\mu} \eta_{\nu}-\eta_{\mu} \partial_{\nu} \hat{g}^{\mu \nu}+\partial_{\nu} \bar{c}_{\mu}\left(\partial_{\rho}\left(\hat{g}^{\mu \nu} c^{\rho}\right)-\hat{g}^{\mu \rho} \partial_{\rho} c^{\nu}-\hat{g}^{\rho \nu} \partial_{\rho} c^{\mu}\right)\right),
$$

which is similar to the action obtained in GR [43]. Hence, the BRST invariant path integral in the $\alpha$-gauge reads

$$
Z_{\mathrm{DUG}}^{\alpha}=\mathcal{N}^{-1} \int \prod_{x} \mathcal{D} g_{\mu \nu} \mathcal{D} \eta_{\mu} \mathcal{D} \bar{c}_{\mu} \mathcal{D} c^{\nu} g^{00}(-g)^{-\frac{3}{2}} \exp \left(\frac{i}{\hbar}\left[S_{E H}\left[g_{\mu \nu}, \Lambda\right]+S_{g f+g h}^{\alpha}\right]\right) .
$$

\subsubsection{Axial gauge}

A well-known condition by computation purposes is the axial gauge. This condition is suitable, in particular, due to the fact that the ghost fields are decoupled and can simply be dropped. It reads

$$
a_{\nu} \hat{g}^{\mu \nu}=0,
$$


where $a_{\nu}$ is a fixed one-form. The gauge and ghost action can be written in the following form

$$
S_{g f+g h}^{A}=\int d^{4} x\left(-a_{(\mu} \eta_{\nu)} \hat{g}^{\mu \nu}-a_{(\mu} \bar{c}_{\nu)}\left[\partial_{\rho}\left(\hat{g}^{\mu \nu} c^{\rho}\right)-\hat{g}^{\mu \rho} \partial_{\rho} c^{\nu}-\hat{g}^{\rho \nu} \partial_{\rho} c^{\mu}\right]\right),
$$

and, finally, we find the path integral in the axial gauge as

$$
Z_{\mathrm{DUG}}^{A}=\mathcal{N}^{-1} \int \prod_{x} \mathcal{D} g_{\mu \nu} \mathcal{D} \eta_{\mu} \mathcal{D} \bar{c}_{\mu} \mathcal{D} c^{\nu} g^{00}(-g)^{-\frac{3}{2}} \exp \left(\frac{i}{\hbar}\left[S_{E H}\left[g_{\mu \nu}, \Lambda\right]+S_{g f+g h}^{A}\right]\right) .
$$

\subsubsection{Planar gauge}

Again, we can consider an extension, the planar gauge, by introducing to the axial gauge an arbitrary constant parameter $\alpha$ such as

$$
a_{\nu} \hat{g}^{\mu \nu}+\alpha \hat{g}_{\mathrm{R}}^{\mu \nu} \eta_{\nu}=0
$$

The limit $\alpha \rightarrow 0$ reproduces the axial gauge. The BRST invariant gauge and ghost action is written in the form

$$
S_{g f+g h}^{P}=\int d^{4} x\left(-\frac{\alpha}{2} \hat{g}_{\mathrm{R}}^{\mu \nu} \eta_{\mu} \eta_{\nu}-a_{(\mu} \eta_{\nu)} \hat{g}^{\mu \nu}-a_{(\mu} \bar{c}_{\nu)}\left[\partial_{\rho}\left(\hat{g}^{\mu \nu} c^{\rho}\right)-\hat{g}^{\mu \rho} \partial_{\rho} c^{\nu}-\hat{g}^{\rho \nu} \partial_{\rho} c^{\mu}\right]\right) .
$$

We thus find the following expression for the path integral in the planar gauge

$$
Z_{\mathrm{DUG}}^{P}=\mathcal{N}^{-1} \int \prod_{x} \mathcal{D} g_{\mu \nu} \mathcal{D} \eta_{\mu} \mathcal{D} \bar{c}_{\mu} \mathcal{D} c^{\nu} g^{00}(-g)^{-\frac{3}{2}} \exp \left(\frac{i}{\hbar}\left[S_{E H}\left[g_{\mu \nu}, \Lambda\right]+S_{g f+g h}^{P}\right]\right) .
$$

\subsubsection{Synchronous gauge}

By means of complementarity let us consider another well-known condition, the synchronous gauge. It reads

$$
\chi_{0}=g_{00}+1=0, \quad \chi_{i}=g_{0 i}=0,
$$

where $i=1,2,3$. We now obtain a non-covariant expression for the gauge and ghost action

$$
\begin{aligned}
S_{g f+g h}^{S}= & \int d^{4} x \sqrt{-g}\left[-\eta^{0}\left(g_{00}+1\right)-\eta^{i} g_{0 i}-\bar{c}^{0} \nabla_{\mu} c^{\mu}\right. \\
& \left.-\bar{c}^{\mu}\left(g_{0 \mu} \nabla_{\nu} c^{\nu}+\partial_{\nu} g_{0 \mu} c^{\nu}+g_{0 \nu} \partial_{\mu} c^{\nu}+g_{\mu \nu} \partial_{0} c^{\nu}\right)\right],
\end{aligned}
$$

where

$$
\nabla_{\mu} c^{\mu}=\partial_{\mu} c^{\mu}+\frac{1}{2} g^{\mu \nu} \partial_{\rho} g_{\mu \nu} c^{\rho}
$$

Finally, the path integral in this gauge is written as

$$
Z_{\mathrm{DUG}}^{S}=\mathcal{N}^{-1} \int \prod_{x} \mathcal{D} g_{\mu \nu} \mathcal{D} \eta_{\mu} \mathcal{D} \bar{c}_{\mu} \mathcal{D} c^{\nu} g^{00}(-g)^{-\frac{3}{2}} \exp \left(\frac{i}{\hbar}\left[S_{E H}\left[g_{\mu \nu}, \Lambda\right]+S_{g f+g h}^{S}\right]\right) .
$$

With this last study we conclude the first analysis by discussing the BRST invariant approach for the DUG theory. This allowed us to determine consistently the respective gauge fixing and ghost action, and subsequently the transition amplitude, for a series of gauge conditions. We shall now extend this study to the UG theory. 


\section{Unimodular gravity with fixed metric determinant}

Once the BRST analysis of the UG theory will resort to subtle points of the Hamiltonian analysis [11], we shall make a brief review of relevant aspects of the Hamiltonian analysis of UG. The standard approach to define UG is to introduce the unimodular condition into Einstein-Hilbert action as a constraint multiplied by a Lagrange multiplier $\lambda$,

$$
S_{\mathrm{UG}}=\int_{\mathcal{M}} d^{4} x\left(\frac{\sqrt{-g} R}{\kappa}-\lambda\left(\sqrt{-g}-\epsilon_{0}\right)\right)+\frac{2}{\kappa} \oint_{\partial \mathcal{M}} d^{3} x \sqrt{|\gamma|} \mathcal{K}
$$

where $\epsilon_{0}$ is a fixed scalar density, such that $\epsilon_{0} d^{4} x$ defines a proper volume element. Then we introduce the ADM variables. The above action is written in ADM form as

$$
S_{\mathrm{UG}}=\int d t \int_{\Sigma_{t}}\left[\frac{N \sqrt{h}}{\kappa}\left(K_{i j} \mathcal{G}^{i j k l} K_{k l}+{ }^{(3)} R\right)-\lambda\left(N \sqrt{h}-\epsilon_{0}\right)\right]+S_{\mathcal{B}}
$$

where $N$ is the lapse variable and $N^{i}$ is the shift vector on the spacelike hypersurface $\Sigma_{t}$, the extrinsic curvature $K_{i j}$ is written as

$$
K_{i j}=\frac{1}{2 N}\left(\partial_{t} h_{i j}-D_{i} N_{j}-D_{j} N_{i}\right),
$$

where $D$ is the covariant derivative that is compatible with the (induced) metric $h_{i j}$ on $\Sigma_{t}$, and $h^{i j}$ is the inverse metric, $h_{i j} h^{j k}=\delta_{i}^{k}$, and the boundary contribution $S_{\mathcal{B}}$ is given as in GR.

The Hamiltonian analysis leads to the following path integral in the $\tilde{\chi}^{\mu}$ gauge condition [11],

$$
\begin{aligned}
Z_{\mathrm{UG}}= & \mathcal{N}^{-1} \int \prod_{x^{\mu}} \mathcal{D} g_{\mu \nu} g^{00}(-g)^{-\frac{3}{2}} \delta\left(\frac{\int_{\Sigma_{t}}\left(\sqrt{-g}-\epsilon_{0}\right)}{\int_{\Sigma_{t}} \sqrt{h}}\right) \\
& \times N \delta\left(\tilde{\chi}^{\mu}\right)\left|\operatorname{det}\left\{\tilde{\chi}^{\mu}, \tilde{\mathcal{H}}_{\nu}\right\}\right| \exp \left(\frac{i}{\hbar} S_{\mathrm{EH}}\left[g_{\mu \nu}\right]\right) .
\end{aligned}
$$

It should be noted that the $\delta$-function imposes the unimodular condition to hold on each slice $\Sigma_{t}$ of spacetime in average, $\int_{\Sigma_{t}}\left(\sqrt{-g}-\epsilon_{0}\right)=0$.

In view of the BRST symmetry, let us recall some subtle points involving the gauge generators of UG. In unimodular gravity with fixed metric determinant, the ADM gauge transformation of a function $\varphi$ of the canonical variables $h_{i j}$ and $\pi^{i j}$ is given as

$$
\delta_{\tilde{\xi}} \varphi=\left\{\varphi, \int_{\Sigma_{t}} \tilde{\mathcal{H}}_{\mu} \tilde{\xi}^{\mu}\right\}, \quad \tilde{\mathcal{H}}_{\mu} \tilde{\xi}^{\mu}=\overline{\mathcal{H}}_{T} \bar{\xi}+\mathcal{H}_{i} \xi^{i}
$$

where the gauge parameter $\tilde{\xi}^{\mu}$ consists of an average-free scalar and a three-vector, $\tilde{\xi}^{\mu}=\left(\bar{\xi}, \xi^{i}\right)$, $\int_{\Sigma_{t}} \sqrt{h} \bar{\xi}=0$, and the generators are the first class (average-free) Hamiltonian and super-momentum constraints $\tilde{\mathcal{H}}_{\mu}=\left(\overline{\mathcal{H}}_{T}, \mathcal{H}_{i}\right)$,

$$
\begin{aligned}
\overline{\mathcal{H}}_{T} & =\overline{\frac{\kappa}{\sqrt{h}} \pi^{i j} \mathcal{G}_{i j k l} \pi^{k l}}-\frac{\overline{\sqrt{h}}(3) R}{\kappa} \approx 0, \\
\mathcal{H}_{i} & =-2 h_{i j} D_{k} \pi^{j k} \approx 0
\end{aligned}
$$


where the overline denotes average-free components, whose integral over space vanish, defined as

$$
\begin{aligned}
\overline{\frac{\kappa}{\sqrt{h}} \pi^{i j} \mathcal{G}_{i j k l} \pi^{k l}} & =\frac{\kappa}{\sqrt{h}} \pi^{i j} \mathcal{G}_{i j k l} \pi^{k l}-\frac{\sqrt{h}}{\int_{\Sigma_{t}} \sqrt{h}} \int_{\Sigma_{t}} \frac{\kappa}{\sqrt{h}} \pi^{i j} \mathcal{G}_{i j k l} \pi^{k l}, \\
\overline{\sqrt{h}(3) R} & =\sqrt{h}^{(3)} R-\frac{\sqrt{h}}{\int_{\Sigma_{t}} \sqrt{h}} \int_{\Sigma_{t}} \sqrt{h}^{(3)} R .
\end{aligned}
$$

Since the zero mode of the Hamiltonian constraint

$$
\mathcal{H}_{0}=\int_{\Sigma_{t}} \mathcal{H}_{T}=\int_{\Sigma_{t}}\left(\frac{\kappa}{\sqrt{h}} \pi^{i j} \mathcal{G}_{i j k l} \pi^{k l}-\frac{\sqrt{h}}{\kappa}(3) R\right)+\lambda_{0} \int_{\Sigma_{t}} \sqrt{h} \approx 0
$$

is a second class constraint in the present theory, it does not generate a gauge transformation.

The average-free gauge parameter $\bar{\xi}$ depends of the metric so that it remains average-free under a variation of the metric,

$$
\delta \int_{\Sigma_{t}} \sqrt{h} \bar{\xi}=\int_{\Sigma_{t}}(\delta \sqrt{h} \bar{\xi}+\sqrt{h} \delta \bar{\xi})=0
$$

This implies that the gauge parameter $\bar{\xi}$ can be expressed as

$$
\bar{\xi}=\xi-\xi_{0}, \quad \xi_{0}=\frac{1}{\int_{\Sigma_{t}} \sqrt{h}} \int_{\Sigma_{t}} \sqrt{h} \xi
$$

where $\xi$ is an unrestricted field that does not depend on any variable. Now the identity $\int_{\Sigma_{t}} \sqrt{h} \bar{\xi}=0$ can be used anywhere, even inside of Poisson brackets. On the other hand, it means that $\bar{\xi}$ has a nonvanishing Poisson bracket with the canonical momentum $\pi^{i j}$.

In the ADM gauge transformation (3.5) we can write the average-free part of the generator as

$$
\int_{\Sigma_{t}} \overline{\mathcal{H}}_{T} \bar{\xi}=\int_{\Sigma_{t}} \mathcal{H}_{T}^{\mathrm{GR}} \xi-\mathcal{H}_{0}^{\mathrm{GR}} \xi_{0}
$$

where $\mathcal{H}_{0}^{\mathrm{GR}}=\int_{\Sigma_{t}} \mathcal{H}_{T}^{\mathrm{GR}}$ and

$$
\mathcal{H}_{T}^{\mathrm{GR}}=\frac{\kappa}{\sqrt{h}} \pi^{i j} \mathcal{G}_{i j k l} \pi^{k l}-\frac{\sqrt{h}}{\kappa}{ }^{(3)} R
$$

Note that $\mathcal{H}_{T}^{\mathrm{GR}}$ and $\mathcal{H}_{0}^{\mathrm{GR}}$ are not constraints in the present theory, since they do not include the cosmological term $\sqrt{h} \lambda_{0}$ (see $(3.10)$ ). Actually, we shall use the following equivalent form of the full generator of the gauge transformations 3.5

$$
\int_{\Sigma_{t}} \tilde{\mathcal{H}}_{\mu} \tilde{\xi}^{\mu}=\int_{\Sigma_{t}}\left(\mathcal{H}_{T}^{\mathrm{GR}} \bar{\xi}+\mathcal{H}_{i} \xi^{i}\right)
$$

since it avoids the appearance of $\mathcal{H}_{0}^{\mathrm{GR}}$ in evaluation of the transformations.

Gauge transformation of canonical variables are obtained from (3.5) as follows. The spatial metric transforms as

$$
\delta_{\tilde{\xi}} h_{i j}=\frac{2 \kappa}{\sqrt{h}} \mathcal{G}_{i j k l} \pi^{k l} \bar{\xi}+\partial_{k} h_{i j} \xi^{k}+h_{i k} \partial_{j} \xi^{k}+h_{k j} \partial_{i} \xi^{k}
$$


since $\left\{h_{i j}, \bar{\xi}\right\}=0$. The canonical momentum $\pi^{i j}$ transforms as

$$
\begin{aligned}
\delta_{\tilde{\xi}} \pi^{i j}= & {\left[\frac{1}{2} h^{i j}\left(\frac{\kappa}{\sqrt{h}} \pi^{k l} \mathcal{G}_{k l m n} \pi^{m n}+\frac{\sqrt{h}}{\kappa}(3) R\right)-\frac{\kappa}{\sqrt{h}}\left(2 \pi_{k}^{(i} \pi^{j) k}-\pi^{i j} h_{k l} \pi^{k l}\right)\right.} \\
& \left.-\frac{\sqrt{h}}{\kappa}\left({ }^{(3)} R^{i j}-D^{i} D^{j}+h^{i j} D^{k} D_{k}\right)\right] \bar{\xi}+\partial_{k}\left(\pi^{i j} \xi^{k}\right)-\pi^{i k} \partial_{k} \xi^{j}-\pi^{k j} \partial_{k} \xi^{i} \\
& +\left(\frac{1}{\int_{\Sigma_{t}} \sqrt{h}} \int_{\Sigma_{t}} \frac{\kappa}{\sqrt{h}} \pi^{i j} \mathcal{G}_{i j k l} \pi^{k l}-\frac{\sqrt{h}}{\kappa}(3) R\right) \frac{1}{2} \sqrt{h} h^{i j} \bar{\xi} .
\end{aligned}
$$

The algebra of gauge transformations is obtained as

$$
\delta_{\tilde{\xi}} \delta_{\tilde{\psi}} \varphi-\delta_{\tilde{\psi}} \delta_{\tilde{\xi}} \varphi=\delta_{[\tilde{\xi}, \tilde{\psi}]} \varphi
$$

where we find the algebra of gauge parameters as

$$
\begin{aligned}
& {[\tilde{\xi}, \tilde{\psi}]^{0}=-\left(\overline{\xi^{i} \partial_{i} \bar{\psi}}-\overline{\partial_{i} \bar{\xi} \psi^{i}}\right),} \\
& {[\tilde{\xi}, \tilde{\psi}]^{i}=-h^{i j}\left(\bar{\xi} \partial_{j} \bar{\psi}-\partial_{j} \bar{\xi} \bar{\psi}\right)-\left(\xi^{j} \partial_{j} \psi^{i}-\partial_{j} \xi^{i} \psi^{j}\right) .}
\end{aligned}
$$

\subsection{BRST Symmetry}

The BRST transformation is obtained as

$$
\begin{aligned}
\delta_{b} h_{i j}= & \left(\frac{2 \kappa}{\sqrt{h}} \mathcal{G}_{i j k l} \pi^{k l} \bar{c}+\partial_{k} h_{i j} c^{k}+h_{i k} \partial_{j} c^{k}+h_{k j} \partial_{i} c^{k}\right) \theta \\
\delta_{b} \pi^{i j}= & {\left[\frac { 1 } { 2 } h ^ { i j } \left(\frac{\kappa}{\sqrt{h}} \pi^{k l} \mathcal{G}_{k l m n} \pi^{m n}+\frac{\sqrt{h}}{\kappa}\left({ }^{3)} R+\frac{\sqrt{h}}{\int_{\Sigma_{t}} \sqrt{h}} \int_{\Sigma_{t}}\left(\frac{\kappa}{\sqrt{h}} \pi^{i j} \mathcal{G}_{i j k l} \pi^{k l}-\frac{\sqrt{h}}{\kappa}{ }^{(3)} R\right)\right)\right.\right.} \\
& \left.-\frac{\kappa}{\sqrt{h}}\left(2 \pi^{(i}{ }_{k} \pi^{j) k}-\pi^{i j} h_{k l} \pi^{k l}\right)-\frac{\sqrt{h}}{\kappa}\left({ }^{(3)} R^{i j}-D^{i} D^{j}+h^{i j} D^{k} D_{k}\right)\right] \bar{c} \theta \\
& +\left(\partial_{k}\left(\pi^{i j} c^{k}\right)-\pi^{i k} \partial_{k} c^{j}-\pi^{k j} \partial_{k} c^{i}\right) \theta, \\
\delta_{b} \bar{c}= & -\frac{1}{2}[\tilde{c}, \tilde{c}]^{0} \theta=\overline{c^{i} \partial_{i} \bar{c}} \theta, \\
\delta_{b} c^{i}= & -\frac{1}{2}[\tilde{c}, \tilde{c}]^{i} \theta=\left(h^{i j} \bar{c} \partial_{j} \bar{c}+c^{j} \partial_{j} c^{i}\right) \theta, \\
\delta_{b} \bar{c}^{*}= & \bar{\eta} \theta, \\
\delta_{b} c_{i}^{*}= & \eta_{i} \theta, \\
\delta_{b} \bar{\eta}= & 0, \\
\delta_{b} \eta_{i}= & 0 .
\end{aligned}
$$

The BRST transformation of metric $h_{i j}$ and the momentum $\pi^{i j}$ are obtained from their gauge transformations (3.16) and (3.17), respectively, by replacing the gauge with parameters as $\bar{\xi} \rightarrow \bar{c} \theta$ and $\xi^{i} \rightarrow c^{i} \theta$. The transformation of the ghosts $\tilde{c}^{\mu}=\left(\bar{c}, c^{i}\right)$ is obtained from the algebra of gauge parameters 3.19 with the same replacement. Since the generator $\overline{\mathcal{H}}_{T}$ has a vanishing integral over space, the ghosts $\bar{c}, \bar{c}^{*}$ and the field $\bar{\eta}$ are average-free as well. 


\subsection{Gauge fixing and ghost action}

As previously stated, the gauge generators in this unimodular setting with fixed metric determinant are the average-free Hamiltonian and super-momentum constraints, $\tilde{\mathcal{H}}_{\mu}=\left(\overline{\mathcal{H}}_{T}, \mathcal{H}_{i}\right)$, demanding that one of the gauge conditions $\tilde{\chi}^{\mu}$ has to be average-free, so that the number of gauge conditions matches the number of generators exactly. We choose it to be the zero-component, since the zero mode of the super-Hamiltonian is a second class constraint, and hence we denote $\tilde{\chi}^{\mu}=\left(\bar{\chi}^{0}, \chi^{i}\right)$.

\subsubsection{Unimodular Faddeev-Popov gauge}

The usual Faddeev-Popov (FP) gauge [4] is defined as

$$
\chi_{\mathrm{FP}}^{0}=\ln h-\Phi \approx 0, \quad \chi_{\mathrm{FP}}^{1}=h_{23} \approx 0, \quad \chi_{\mathrm{FP}}^{2}=h_{31} \approx 0, \quad \chi_{\mathrm{FP}}^{3}=h_{12} \approx 0,
$$

where $\ln h=\ln \left(\operatorname{det} h_{i j}\right)$ and $\Phi$ is a fixed function. The average-free component $\overline{\ln h}$ of $\ln h$ is not a scalar density of any weight. Hence it is unclear which measure we should use to integrate $\ln h$ over $\Sigma_{t}$. Here we treat $\ln h$ as a scalar, so that

$$
\overline{\ln h}=\ln h-\frac{1}{\int_{\Sigma_{t}} \sqrt{h}} \int_{\Sigma_{t}} \sqrt{h} \ln h
$$

The unimodular FP gauge conditions are thus defined as

$$
\bar{\chi}_{\mathrm{FP}}^{0}=\overline{\ln h}-\bar{\Phi} \approx 0, \quad \chi_{\mathrm{FP}}^{i}=\frac{1}{2} d^{i j k} h_{j k} \approx 0,
$$

where $\bar{\Phi}$ is a function with zero average, $\int_{\Sigma_{t}} \sqrt{h} \bar{\Phi}=0$, and the last three conditions $\chi_{\mathrm{FP}}^{i}(i=1,2,3)$ are identical to those in (3.21), which impose the off-diagonal components of the metric to vanish, but written with the help of a strictly positive permutation symbol

$$
d^{i j k}= \begin{cases}1, & \text { if the indices } i j k \text { are any permutation of } 123 \\ 0, & \text { if any of the indices } i j k \text { are equal. }\end{cases}
$$

The BRST invariant gauge and ghost action in the unimodular FP gauge is given by

$$
\begin{aligned}
S_{g f+g h}^{\mathrm{FP}}= & \int d^{4} x\left(-\sqrt{h} \bar{\eta}(\overline{\ln h}-\bar{\Phi})-\frac{1}{2} \sqrt{h} \eta_{i} d^{i j k} h_{j k}\right. \\
& -\sqrt{h} \bar{c}^{*}(\overline{\ln h}-\bar{\Phi}+2)\left(K \bar{c}+D_{i} c^{i}\right)-\sqrt{h} c_{i}^{*} d^{i j k}\left(K_{j k}+\frac{1}{2} h_{j k} K\right) \bar{c} \\
& \left.-\frac{1}{2} \sqrt{h} c_{i}^{*} d^{i j k}\left(h_{j k} D_{l} c^{l}+\partial_{l} h_{j k} c^{l}+h_{j l} \partial_{k} c^{l}+h_{l k} \partial_{j} c^{l}\right)\right) .
\end{aligned}
$$

It should be noted that 3.25 is written in terms of the extrinsic curvature $K_{i j}$ and not momentum $\pi^{i j}$. This is because when the canonical momenta are integrated in the path integral, the momentum $\pi^{i j}$ is expressed in terms of the metric variables as

$$
\pi^{i j}=\frac{\sqrt{h}}{\kappa} \mathcal{G}^{i j k l} K_{k l}
$$


Moreover, in obtaining (3.25), we used the fact that the (average-free) ghost $\bar{c}^{*}$ has a vanishing average, so that for any time-dependent function $f(t)$ we obtain $\int d^{4} x \bar{c}^{*} \sqrt{h} f(t)=0$.

The path integral for the unimodular Faddeev-Popov gauge condition is written as

$$
\begin{aligned}
Z_{\mathrm{UG}}^{\mathrm{FP}}= & \mathcal{N}^{-1} \int \prod_{x} \mathcal{D} g_{\mu \nu} \mathcal{D} \bar{\eta} \mathcal{D} \eta_{i} \mathcal{D} \bar{c}^{*} \mathcal{D} \bar{c} \mathcal{D} c_{i}^{*} \mathcal{D} c^{j} g^{00}(-g)^{-\frac{3}{2}} \\
& \times \delta\left(\frac{\int_{\Sigma_{t}}\left(\sqrt{-g}-\epsilon_{0}\right)}{\left(-g^{00}\right)^{-\frac{1}{2}} \int_{\Sigma_{t}} \sqrt{h}}\right) \exp \left(\frac{i}{\hbar}\left[S_{E H}\left[g_{\mu \nu}\right]+S_{g f+g h}^{F P}\right]\right) .
\end{aligned}
$$

\subsubsection{Averaged metric determinant and spatial harmonic gauge}

To illustrate the analysis with further examples we consider now a mixed unimodular condition. The first (average-free) gauge condition is chosen to agree with the unimodular Faddeev-Popov gauge (3.23), while the other conditions define harmonic coordinates on each spatial hypersurface $\Sigma_{t}$ :

$$
\bar{\chi}_{\mathrm{FP}}^{0}=\overline{\ln h}-\bar{\Phi} \approx 0, \quad \chi_{\mathrm{H}}^{i}=\partial_{j}\left(\sqrt{h} h^{i j}\right) \approx 0 .
$$

The BRST invariant gauge and ghost action reads

$$
\begin{aligned}
S_{g f+g h}^{\mathrm{DH}}= & \int d^{4} x\left(-\sqrt{h} \bar{\eta}(\overline{\ln h}-\bar{\Phi})-\eta_{i} \partial_{j}\left(\sqrt{h} h^{i j}\right)-\sqrt{h} \bar{c}^{*}(\overline{\ln h}-\bar{\Phi}+2)\left(K \bar{c}+D_{i} c^{i}\right)\right. \\
& +2 c_{i}^{*} \partial_{j}\left[\sqrt{h}\left(K^{i j}-\frac{1}{2} h^{i j} K\right) \bar{c}\right]-c_{i}^{*} \partial_{j} \partial_{k}\left(\sqrt{h} h^{i k}\right) c^{j} \\
& \left.-c_{i}^{*} \partial_{j}\left(\sqrt{h} h^{i j}\right) \partial_{k} c^{k}+c_{i}^{*} \partial_{j}\left(\sqrt{h} h^{j k}\right) \partial_{k} c^{i}+c_{i}^{*} \sqrt{h} h^{j k} \partial_{j} \partial_{k} c^{i}\right) .
\end{aligned}
$$

once again the momentum has been expressed in terms of metric variables (3.26), and we denote $K^{i j}=h^{i k} h^{j l} K_{k l}$. Finally, the path integral is given as

$$
\begin{aligned}
Z_{\mathrm{UG}}^{\mathrm{DH}}= & \mathcal{N}^{-1} \int \prod_{x} \mathcal{D} g_{\mu \nu} \mathcal{D} \bar{\eta} \mathcal{D} \eta_{i} \mathcal{D} \bar{c}^{*} \mathcal{D} \bar{c} \mathcal{D} c_{i}^{*} \mathcal{D} c^{j} g^{00}(-g)^{-\frac{3}{2}} \\
& \times \delta\left(\frac{\int_{\Sigma_{t}}\left(\sqrt{-g}-\epsilon_{0}\right)}{\left(-g^{00}\right)^{-\frac{1}{2}} \int_{\Sigma_{t}} \sqrt{h}}\right) \exp \left(\frac{i}{\hbar}\left[S_{E H}\left[g_{\mu \nu}\right]+S_{g f+g h}^{D H}\right]\right) .
\end{aligned}
$$

\subsubsection{Averaged metric trace and spatial harmonic gauge}

Another alternative gauge condition is proposed as: the first gauge condition is chosen to be the average-free component of the trace of the spatial metric, while the other conditions define harmonic coordinates on each spatial hypersurface $\Sigma_{t}$ :

$$
\bar{\chi}_{\mathrm{T}}^{0}=\overline{\operatorname{tr}\left(h_{i j}\right)} \approx 0, \quad \chi_{\mathrm{H}}^{i}=\partial_{j}\left(\sqrt{h} h^{i j}\right) \approx 0,
$$

where

$$
\overline{\operatorname{tr}\left(h_{i j}\right)}=\operatorname{tr}\left(h_{i j}\right)-\frac{1}{\int_{\Sigma_{t}} \sqrt{h}} \int_{\Sigma_{t}} \sqrt{h} \operatorname{tr}\left(h_{i j}\right) ; \quad \operatorname{tr}\left(h_{i j}\right)=\sum_{i} h_{i i} .
$$


Hence, the BRST invariant gauge and ghost action in the trace gauge condition is found to be

$$
\begin{aligned}
S_{g f+g h}^{\mathrm{TH}}= & \int d^{4} x\left(-\sqrt{h} \bar{\eta} \overline{\operatorname{tr}\left(h_{i j}\right)}-\eta_{i} \partial_{j}\left(\sqrt{h} h^{i j}\right)-\sqrt{h} \bar{c}^{*} \overline{\operatorname{tr}\left(h_{i j}\right)}\left(K \bar{c}+D_{i} c^{i}\right)\right. \\
& -\sqrt{h} \bar{c}^{*} \sum_{i}\left(2 K_{i i} \bar{c}+\partial_{j} h_{i i} c^{j}+2 h_{i j} \partial_{i} c^{j}\right)+2 c_{i}^{*} \partial_{j}\left[\sqrt{h}\left(K^{i j}-\frac{1}{2} h^{i j} K\right) \bar{c}\right] \\
& \left.-c_{i}^{*} \partial_{j} \partial_{k}\left(\sqrt{h} h^{i k}\right) c^{j}-c_{i}^{*} \partial_{j}\left(\sqrt{h} h^{i j}\right) \partial_{k} c^{k}+c_{i}^{*} \partial_{j}\left(\sqrt{h} h^{j k} \partial_{k} c^{i}\right)\right) .
\end{aligned}
$$

Finally, the path integral is written as

$$
\begin{aligned}
Z_{\mathrm{UG}}^{\mathrm{TH}}= & \mathcal{N}^{-1} \int \prod_{x} \mathcal{D} g_{\mu \nu} \mathcal{D} \bar{\eta} \mathcal{D} \eta_{i} \mathcal{D} \bar{c}^{*} \mathcal{D} \bar{c} \mathcal{D} c_{i}^{*} \mathcal{D} c^{j} g^{00}(-g)^{-\frac{3}{2}} \\
& \times \delta\left(\frac{\int_{\Sigma_{t}}\left(\sqrt{-g}-\epsilon_{0}\right)}{\left(-g^{00}\right)^{-\frac{1}{2}} \int_{\Sigma_{t}} \sqrt{h}}\right) \exp \left(\frac{i}{\hbar}\left[S_{E H}\left[g_{\mu \nu}\right]+S_{g f+g h}^{\mathrm{TH}}\right]\right) .
\end{aligned}
$$

Before concluding this section, we mention a problem that can appear in the present theory if one uses a (average-free) gauge condition that involves the canonical momentum $\pi^{i j}$. In particular, adapting the usual Dirac gauge conditions to the present unimodular theory with fixed metric determinant involves a problem which is discussed in Appendix A.

With this section we conclude the first part of our analysis by discussing the BRST invariant approach for the DUG and UG theory. We have determined the BRST invariant path integral for both theories for a set of gauge conditions. We now proceed further and extend the previous study by establishing connections between transition amplitude in different gauges. To achieve this goal, we shall first introduce the finite field-dependent BRST transformations.

\section{The Generalized BRST transformation}

In this section, we illustrate the FFBRST (generalized BRST) formulation [16 for the unimodular gravity theory with full diffeomorphism invariance in an elegant way. For that matter, we first write the BRST transformation for all the fields of the theory, Eqs.2.10a)-(2.10d), denoted collectively as $\phi_{a}(x) \equiv \phi(x)$, as follows:

$$
\phi(x) \longrightarrow \phi^{\prime}(x)=\phi(x)+s_{b} \phi(x) \theta,
$$

where $s_{b} \phi$ is the Slavnov variation of the field $\phi(x)$ and $\theta$ is a Grassmann global parameter.

To generalize the BRST symmetry, we first make all the fields $\phi(x)$ depend on a continuous parameter $\kappa(0 \leq \kappa \leq 1)$ in such a way that the conditions $\phi(x, \kappa=0) \equiv \phi(x)$ and $\phi(x, \kappa=$ $1) \equiv \phi^{\prime}(x)=\phi(x)+s_{b} \phi(x) \theta[\phi]$ stand for the original field and the FFBRST transformed field, respectively, where $\theta[\phi]$ is now a (functional) finite field-dependent parameter. Moreover, the FFBRST 
transformation is justified by the following infinitesimal field-dependent BRST transformation:

$$
\begin{aligned}
\frac{d g_{\mu \nu}(x, \kappa)}{d \kappa} & =\left(\partial_{\rho} g_{\mu \nu} c^{\rho}+g_{\mu \rho} \partial_{\nu} c^{\rho}+g_{\rho \nu} \partial_{\mu} c^{\rho}\right) \theta^{\prime}[\phi(\kappa)] \\
\frac{d c^{\mu}(x, \kappa)}{d \kappa} & =-c^{\nu} \partial_{\nu} c^{\mu} \theta^{\prime}[\phi(\kappa)] \\
\frac{d \bar{c}_{\mu}(x, \kappa)}{d \kappa} & =\eta_{\mu} \theta^{\prime}[\phi(\kappa)] \\
\frac{d \eta_{\mu}(x, \kappa)}{d \kappa} & =0
\end{aligned}
$$

Integrating these equations with respect to $\kappa$, we find the following field-dependent transformations

$$
\begin{aligned}
g_{\mu \nu}(x, \kappa) & =g_{\mu \nu}(x, 0)+\left(\partial_{\rho} g_{\mu \nu} c^{\rho}+g_{\mu \rho} \partial_{\nu} c^{\rho}+g_{\rho \nu} \partial_{\mu} c^{\rho}\right) \theta[\phi(\kappa)] \\
c^{\mu}(x, \kappa) & =c^{\mu}(x, 0)-c^{\nu} \partial_{\nu} c^{\mu} \theta[\phi(\kappa)] \\
\bar{c}_{\mu}(x, \kappa) & =\bar{c}_{\mu}(x, 0)+\eta_{\mu} \theta[\phi(\kappa)] \\
\eta_{\mu}(x, \kappa) & =0
\end{aligned}
$$

where we have $\theta[\phi(\kappa)]$ as a functional of the fields $\phi(x, \kappa)[16$

$$
\begin{aligned}
\theta[\phi(\kappa)] & =\int_{0}^{\kappa} d \kappa \theta^{\prime}[\phi(\kappa)] \\
& =\theta^{\prime}[\phi(0)] \frac{\exp \left(\kappa \frac{\delta \theta^{\prime}}{\delta \phi} s_{b} \phi\right)-1}{\frac{\delta \theta^{\prime}}{\delta \phi} s_{b} \phi} .
\end{aligned}
$$

At the boundary value of $\kappa$, i.e. $\kappa=1$, these expressions yield to the FFBRST transformations,

$$
\begin{aligned}
\delta_{b} g_{\mu \nu}(x) & =\left(\partial_{\rho} g_{\mu \nu} c^{\rho}+g_{\mu \rho} \partial_{\nu} c^{\rho}+g_{\rho \nu} \partial_{\mu} c^{\rho}\right) \theta[\phi(1)] \\
\delta_{b} c^{\mu}(x) & =-c^{\nu} \partial_{\nu} c^{\mu} \theta[\phi(1)] \\
\delta_{b} \bar{c}_{\mu}(x) & =\eta_{\mu} \theta[\phi(1)] \\
\delta_{b} \eta_{\mu}(x) & =0
\end{aligned}
$$

where finite field-dependent parameter reads $\theta[\phi(1)]=\theta[\phi(\kappa)]_{\kappa=1}$.

Here we notice that the resulting FFBRST transformations with field-dependent parameter 4.5 are a symmetry of the effective action. However, the path integral measure changes non-trivially under these leading thus to a non-trivial Jacobian. Hence, it is necessary derive the explicit expression of the Jacobian for the functional measure under the FFBRST transformations for an arbitrary $\theta$ parameter.

\subsection{Jacobian for field-dependent BRST transformation}

To compute the Jacobian we first define the path integral for unimodular gravity theory in a general gauge as follows,

$$
Z=\int \mathcal{D} \Phi e^{\left(\frac{i}{\hbar} S_{E H}[\phi]+S_{g f+g h}[\phi]\right)}
$$


where $\mathcal{D} \Phi$ is the (BRST) covariant functional measure and $S_{g f+g h}[\phi]$ refers to the general gauge-fixing and ghost part of the effective action. In order to determine the Jacobian expression for the functional measure under the FFBRST transformations, we write 16

$$
\mathcal{D} \Phi(\kappa)=J(\kappa) \mathcal{D} \Phi(\kappa)=J(\kappa+d \kappa) \mathcal{D} \Phi(\kappa+d \kappa) .
$$

Since the transformation from $\phi(\kappa)$ to $\phi(\kappa+d \kappa)$ is viewed as an infinitesimal one, this can further be written as $[16$

$$
\frac{J(\kappa)}{J(\kappa+d \kappa)}=\sum_{\phi} \pm \frac{\delta \phi(\kappa+d \kappa)}{\delta \phi(\kappa)}
$$

where \pm sign is used for bosonic and fermionic fields, respectively. Now, upon Taylor expansion the above expression yields

$$
1-\frac{1}{J} \frac{d J}{d \kappa} d \kappa=1+d \kappa \int d^{4} x \sum_{\phi} \pm s_{b} \phi(x, \kappa) \frac{\delta \theta^{\prime}[\phi(\kappa)]}{\delta \phi(\kappa)},
$$

which further simplifies to

$$
\frac{d \ln J[\phi]}{d \kappa}=-\int d^{4} x \sum_{\phi} \pm s_{b} \phi(x, \kappa) \frac{\delta \theta^{\prime}[\phi(\kappa)]}{\delta \phi(\kappa)} .
$$

We now perform the integration over $\kappa$ (after Taylor expansion) with an appropriate limit, to get the following:

$$
\begin{aligned}
\ln J[\phi] & =-\int_{0}^{1} d \kappa \int d^{4} x \sum_{\phi} \pm s_{b} \phi(x, \kappa) \frac{\delta \theta^{\prime}[\phi(\kappa)]}{\delta \phi(\kappa)} \\
& =-\left(\int d^{4} x \sum_{\phi} \pm s_{b} \phi(x) \frac{\delta \theta^{\prime}[\phi]}{\delta \phi}\right)
\end{aligned}
$$

This result leads to the final expression for the Jacobian generated from a variation of the functional measure under FFBRST transformations with an arbitrary parameter

$$
J[\phi]=\exp \left(-\int d^{4} x \sum_{\phi} \pm s_{b} \phi(x) \frac{\delta \theta^{\prime}[\phi]}{\delta \phi}\right) .
$$

We remark here that this expression of Jacobian is rather elegant than one originally derived in [16]. Since the Jacobian obtained here depends explicitly on the parameter $\theta^{\prime}$.

Now, with the expression (4.12 for the Jacobian (generated by FFBRST transformation) we find that the path integral 4.6 changes as

$$
\begin{aligned}
\int \mathcal{D} \Phi^{\prime} e^{\left(\frac{i}{\hbar} S_{E H}\left[\phi^{\prime}\right]+S_{g f+g h}\left[\phi^{\prime}\right]\right)} & =\int J[\phi] \mathcal{D} \Phi e^{\left(\frac{i}{\hbar} S_{E H}[\phi]+S_{g f+g h}[\phi]\right)} \\
& =\int \mathcal{D} \Phi e^{\left(\frac{i}{\hbar} S_{E H}[\phi]+S_{g f+g h}[\phi]-\int d^{4} x\left(\sum_{\phi} \pm s_{b} \phi \frac{\delta \theta^{\prime}}{\delta \phi}\right)\right)} .
\end{aligned}
$$


This is the FFBRST transformed path integral of the unimodular gravity theories (both DUG and UG) with an extended action, where the gauge fixing and ghosts actions are modified by the Jacobian. We emphasize that the form of the functional parameter $\theta^{\prime}$ should be chosen so that the Jacobian (4.12 does not produce any physical change in the quantum theory. Otherwise, one could choose $\theta^{\prime}$ so that the physical content of the quantum theory is modified, e.g. producing new vertices and/or propagating modes, which would not be a symmetry transformation. For this matter we emphasize that we consider in our analysis only the path integral of the vacuum transition amplitude. We shall now illustrate this result by establishing the connection between different gauges of the two presented versions of unimodular gravity.

\subsection{Connection of different gauges in fully diffeomorphism-invariant theory}

In this section we study the connection of various important gauges of the fully diffeomorphisminvariant unimodular gravity (as stated in section 2). In particular, notice that these are well-defined gauges, since then there should be no physical change in the quantum theory. We will show the connection between the following gauges: (i) harmonic and synchronous gauges, (ii) axial and harmonic gauges, (iii) harmonic and Lorentz gauges, and, at last, (iv) Lorentz and synchronous gauges.

\subsubsection{Harmonic to synchronous gauge}

For this analysis, we follow the standard procedure as discussed above. We first construct the infinitesimal version of the functional parameter (4.5) as follows

$$
\theta^{\prime}[\phi]=-\int d^{4} x\left[-\bar{c}_{\mu} \partial_{\nu} \hat{g}^{\mu \nu}+\sqrt{-g} \bar{c}^{0}\left(g_{00}+1\right)+\sqrt{-g} \bar{c}^{i} g_{0 i}\right]
$$

The advantage of constructing an infinitesimal version is that with such parameter the Jacobian can be computed directly from (4.12). Thus, the Jacobian expression for this choice of parameter (4.14) is

$$
\begin{aligned}
J[\phi]= & \exp \left[\int d ^ { 4 } x \left(\eta_{\mu} \partial_{\nu} \hat{g}^{\mu \nu}+\bar{c}_{\mu}\left[\partial_{\nu}\left(\partial_{\rho}\left(\hat{g}^{\mu \nu} c^{\rho}\right)-\hat{g}^{\mu \rho} \partial_{\rho} c^{\nu}-\hat{g}^{\rho \nu} \partial_{\rho} c^{\mu}\right)\right]\right.\right. \\
& -\sqrt{-g} \eta^{0}\left(g_{00}+1\right)-\sqrt{-g} \eta^{i} g_{0 i}-\sqrt{-g} \bar{c}^{0} \nabla_{\mu} c^{\mu} \\
& \left.\left.-\sqrt{-g} \bar{c}^{\mu}\left(g_{0 \mu} \nabla_{\nu} c^{\nu}+\partial_{\nu} g_{0 \mu} c^{\nu}+g_{0 \nu} \partial_{\mu} c^{\nu}+g_{\mu \nu} \partial_{0} c^{\nu}\right)\right)\right] .
\end{aligned}
$$

With this Jacobian the generating functional in harmonic gauge 2.15 changes to

$$
\begin{aligned}
\mathcal{N}^{-1} \int \mathcal{D} \Phi^{\prime} e^{i\left(S_{E H}\left[\phi^{\prime}\right]+S_{g f+g h}^{H}\left[\phi^{\prime}\right]\right)} & =\mathcal{N}^{-1} \int J[\phi] \mathcal{D} \Phi e^{i\left(S_{E H}[\phi]+S_{g f+g h}^{H}[\phi]\right)} \\
& =\mathcal{N}^{-1} \int \mathcal{D} \Phi e^{i\left(S_{E H}[\phi]+S_{g f+g h}^{S}[\phi]\right)} \\
& =Z_{\mathrm{DUG}}^{S}
\end{aligned}
$$

which is nothing but the transition amplitude in synchronous gauge 2.18). Here $\phi^{\prime}$ and $\phi$ denote, respectively, the transformed and generic fields of the DUG theory. The invariant functional measure for DUG is defined as $\mathcal{D} \Phi \equiv \prod_{x} \mathcal{D} g_{\mu \nu} \mathcal{D} \eta_{\mu} \mathcal{D} \bar{c}_{\mu} \mathcal{D} c^{\nu} g^{00}(-g)^{-\frac{3}{2}}$. Thus the FFBRST transformation with parameter 4.14 establishes the connection between harmonic and synchronous gauges, Eqs. (2.13) and 2.25, respectively, for fully diffeomorphism-invariant unimodular gravity theory. 


\subsubsection{Axial to harmonic gauge}

To relate axial and harmonic gauges, Eqs.2.19) and (2.13), respectively, we consider the following infinitesimal field-dependent parameter

$$
\theta^{\prime}[\phi]=-\int d^{4} x\left[-a_{(\mu} \eta_{\nu)} \hat{g}^{\mu \nu}+\bar{c}_{\mu} \partial_{\nu} \hat{g}^{\mu \nu}\right] .
$$

The Jacobian for functional measure under FFBRST transformation is calculated by

$$
\begin{aligned}
J[\phi]= & \exp \left[\int d ^ { 4 } x \left(a_{(\mu} \eta_{\nu)} \hat{g}^{\mu \nu}+a_{(\mu} \bar{c}_{\nu)}\left[\partial_{\rho}\left(\hat{g}^{\mu \nu} c^{\rho}\right)-\hat{g}^{\mu \rho} \partial_{\rho} c^{\nu}-\hat{g}^{\rho \nu} \partial_{\rho} c^{\mu}\right]\right.\right. \\
& \left.\left.-\eta_{\mu} \partial_{\nu} \hat{g}^{\mu \nu}+\bar{c}_{\mu}\left[-\partial_{\nu}\left(\partial_{\rho}\left(\hat{g}^{\mu \nu} c^{\rho}\right)-\hat{g}^{\mu \rho} \partial_{\rho} c^{\nu}-\hat{g}^{\rho \nu} \partial_{\rho} c^{\mu}\right)\right]\right)\right] .
\end{aligned}
$$

Now substituting this Jacobian (4.18) into the expression of path integral measure in axial gauge (2.21) as follows

$$
\begin{aligned}
\mathcal{N}^{-1} \int \mathcal{D} \Phi^{\prime} e^{i\left(S_{E H}\left[\phi^{\prime}\right]+S_{g f+g h}^{A}\left[\phi^{\prime}\right]\right)} & =\mathcal{N}^{-1} \int J[\phi] \mathcal{D} \Phi e^{i\left(S_{E H}[\phi]+S_{g f+g h}^{A}[\phi]\right)} \\
& =\mathcal{N}^{-1} \int \mathcal{D} \Phi e^{i\left(S_{E H}[\phi]+S_{g f+g h}^{H}[\phi]\right)} \\
& =Z_{\mathrm{DUG}}^{H}
\end{aligned}
$$

and we thus get the expression of path integral in harmonic gauge (2.15). Therefore, FFBRST transformation, generated with the parameter (4.17), connects the axial and harmonic gauges of the theory.

Here we remark that the same value of Jacobian given in (4.18) when replaced into the expression of the transition amplitude in Lorentz gauge (2.18) gives the transition amplitude in planar gauge (2.24). Thus, the FFBRST transformation with parameter 4.17) also connects the Lorentz gauge (2.16) to planar gauge 2.22).

\subsubsection{Harmonic to Lorentz gauge}

To establish the connection of the harmonic gauge to Lorentz gauge, Eqs.2.13) and (2.16), respectively, we determine the infinitesimal functional parameter as follows

$$
\theta^{\prime}[\phi]=-\int d^{4} x\left[\bar{c}_{\mu} \frac{\alpha}{2} \hat{g}_{R}^{\mu \nu} \eta_{\nu}\right]
$$

Utilizing this parameter the Jacobian for path integral measure is calculated by

$$
J[\phi]=\exp \left[\int d^{4} x\left(-\frac{\alpha}{2} \hat{g}_{\mathrm{R}}^{\mu \nu} \eta_{\mu} \eta_{\nu}\right)\right] .
$$

This value for the Jacobian when inserted into the transition amplitude changes the theory from the harmonic gauge 2.15) into the one in the Lorentz gauge 2.18) as follows

$$
\begin{aligned}
\mathcal{N}^{-1} \int \mathcal{D} \Phi^{\prime} e^{i\left(S_{E H}\left[\phi^{\prime}\right]+S_{g f+g h}^{H}\left[\phi^{\prime}\right]\right)} & =\mathcal{N}^{-1} \int J[\phi] \mathcal{D} \phi e^{i\left(S_{E H}[\phi]+S_{g f+g h}^{H}[\phi]\right)} \\
& =\mathcal{N}^{-1} \int \mathcal{D} \phi e^{i\left(S_{E H}[\phi]+S_{g f+g h}^{L}[\phi]\right)} \\
& =Z_{\mathrm{DUG}}^{L}
\end{aligned}
$$


Here we emphasize that the Jacobian expression (4.21) is also responsible to connect the axial gauge (2.19) to planar gauge (2.22). Thus the path integral for DUG in axial gauge (2.21) under FFBRST transformation with parameter 4.20 switches to the transition amplitude in planar gauge 2.24 .

\subsubsection{Lorentz to synchronous gauge}

Finally, we determine the connection between Lorentz gauge and synchronous gauge, Eqs. (2.16) and 2.25), respectively. For this purpose we construct the functional parameter as follows

$$
\theta^{\prime}[\phi]=-\int d^{4} x\left[-\bar{c}_{\mu} \frac{\alpha}{2} \hat{g}_{R}^{\mu \nu} \eta_{\nu}-\bar{c}_{\mu} \partial_{\nu} \hat{g}^{\mu \nu}+\sqrt{-g} \bar{c}^{0}\left(g_{00}+1\right)+\sqrt{-g} \bar{c}^{i} g_{0 i}\right] .
$$

The corresponding Jacobian is found to read

$$
\begin{aligned}
J[\phi]= & \exp \left[\int d ^ { 4 } x \left(\frac{\alpha}{2} \hat{g}_{\mathrm{R}}^{\mu \nu} \eta_{\mu} \eta_{\nu}+\eta_{\mu} \partial_{\nu} \hat{g}^{\mu \nu}+\bar{c}_{\mu}\left[\partial_{\nu}\left(\partial_{\rho}\left(\hat{g}^{\mu \nu} c^{\rho}\right)-\hat{g}^{\mu \rho} \partial_{\rho} c^{\nu}-\hat{g}^{\rho \nu} \partial_{\rho} c^{\mu}\right)\right]\right.\right. \\
& -\sqrt{-g} \eta^{0}\left(g_{00}+1\right)-\sqrt{-g} \eta^{i} g_{0 i}-\sqrt{-g} \bar{c}^{0} \nabla_{\mu} c^{\mu} \\
& \left.\left.-\sqrt{-g} \bar{c}^{\mu}\left(g_{0 \mu} \nabla_{\nu} c^{\nu}+\partial_{\nu} g_{0 \mu} c^{\nu}+g_{0 \nu} \partial_{\mu} c^{\nu}+g_{\mu \nu} \partial_{0} c^{\nu}\right)\right)\right] .
\end{aligned}
$$

Substituting this value (4.24) into the generating functional in Lorentz gauge 2.18 we get

$$
\begin{aligned}
\mathcal{N}^{-1} \int \mathcal{D} \Phi^{\prime} e^{i\left(S_{E H}\left[\phi^{\prime}\right]+S_{g f+g h}^{\alpha}\left[\phi^{\prime}\right]\right)} & =\mathcal{N}^{-1} \int J[\phi] \mathcal{D} \Phi e^{i\left(S_{E H}[\phi]+S_{g f+g h}^{\alpha}[\phi]\right)} \\
& =\mathcal{N}^{-1} \int \mathcal{D} \Phi e^{i\left(S_{E H}[\phi]+S_{g f+g h}^{S}[\phi]\right)} \\
& =Z_{\mathrm{DUG}}^{S} .
\end{aligned}
$$

This establishes a connection between the path integral on Lorentz gauge (2.18) and synchronous gauge 2.28).

Hence we concluded this subsection of analysis of FFBRST equivalence by establishing relations among different and relevant gauge conditions of fully-diffeomorphism invariant theory of unimodular gravity. Next we will perform a similar analysis but now for unimodular gravity with fixed metric determinant.

\subsection{Connection of different gauges in unimodular gravity with fixed metric deter- minant}

In this subsection we analyse the connection of different gauges of unimodular gravity with fixed metric determinant. Following the results from section3, the FFBRST transformation for unimodular gravity with fixed metric determinant are determined by the replacement of the parameter $\theta \rightarrow \theta[\phi]$ into the Eqs. 3.20a - 3.20h).

With these results we will show the following mapping: (i) unimodular Faddeev-Popov to averaged metric determinant and spatial harmonic gauges, (ii) unimodular Faddeev-Popov to averaged metric trace and spatial harmonic gauges, and, finally, (iii) averaged metric determinant to averaged metric trace gauges. 


\subsubsection{Unimodular Faddeev-Popov to averaged metric determinant and spatial harmonic gauges}

In order to map the unimodular Faddeev-Popov and averaged metric determinant and spatial harmonic gauges, Eqs. 3.23) and (3.28), respectively, we define the infinitesimal field-dependent parameter as follows

$$
\theta^{\prime}[\phi]=-\int d^{4} x\left[-\frac{1}{2} c_{i}^{*} \sqrt{h} d^{i j k} h_{j k}+c_{i}^{*} \partial_{j}\left(\sqrt{h} h^{i j}\right)\right] .
$$

Now with the help of expression 4.12 we compute the respective Jacobian corresponding to this parameter

$$
\begin{aligned}
J[\phi]= & \exp \left[\int d ^ { 4 } x \left(\frac{1}{2} \sqrt{h} \eta_{i} d^{i j k} h_{j k}+\sqrt{h} c_{i}^{*} d^{i j k}\left(K_{j k}+\frac{1}{2} h_{j k} K\right) \bar{c}\right.\right. \\
& +\frac{1}{2} \sqrt{h} c_{i}^{*} d^{i j k}\left(h_{j k} D_{l} c^{l}+\partial_{l} h_{j k} c^{l}+h_{j l} \partial_{k} c^{l}+h_{l k} \partial_{j} c^{l}\right)-\eta_{i} \partial_{j}\left(\sqrt{h} h^{i j}\right) \\
& +2 c_{i}^{*} \partial_{j}\left[\sqrt{h}\left(K^{i j}-\frac{1}{2} h^{i j} K\right) \bar{c}\right]-c_{i}^{*} \partial_{j} \partial_{k}\left(\sqrt{h} h^{i k}\right) c^{j}-c_{i}^{*} \partial_{j}\left(\sqrt{h} h^{i j}\right) \partial_{k} c^{k} \\
& \left.\left.+c_{i}^{*} \partial_{j}\left(\sqrt{h} h^{j k}\right) \partial_{k} c^{i}+c_{i}^{*} \sqrt{h} h^{j k} \partial_{j} \partial_{k} c^{i}\right)\right] .
\end{aligned}
$$

With this result for the Jacobian 4.27) the transition amplitude for unimodular gravity with fixed metric determinant in Faddeev-Popov gauge (3.27) changes as

$$
\begin{aligned}
\mathcal{N}^{-1} \int \mathcal{D} \Phi^{\prime} e^{\frac{i}{\hbar}\left(S_{E H}\left[\phi^{\prime}\right]+S_{g f+g h}^{F P}\right)} & =\mathcal{N}^{-1} \int J[\phi] \mathcal{D} \Phi e^{\frac{i}{\hbar}\left(S_{E H}[\phi]+S_{g f+g h}^{F P}\right)} \\
& =\mathcal{N}^{-1} \int \mathcal{D} \Phi e^{\frac{i}{\hbar}\left(S_{E H}[\phi]+S_{g f+g h}^{D H}\right)} \\
& =Z_{\mathrm{UG}}^{D H}
\end{aligned}
$$

which is exactly the expression for the path integral in averaged metric determinant and spatial harmonic gauge. Here the explicit expression for the invariant functional measure is now given as, $\mathcal{D} \Phi \equiv \prod_{x} \mathcal{D} g_{\mu \nu} \mathcal{D} \bar{\eta} \mathcal{D} \eta_{i} \mathcal{D} \bar{c}^{*} \mathcal{D} \bar{c} \mathcal{D} c_{i}^{*} \mathcal{D} c^{j} g^{00}(-g)^{-\frac{3}{2}} \delta\left(\frac{\int_{\Sigma_{t}}\left(\sqrt{-g}-\epsilon_{0}\right)}{\left(-g^{00}\right)^{-\frac{1}{2}} \int_{\Sigma_{t}} \sqrt{h}}\right)$.

\subsubsection{Unimodular Faddeev-Popov to averaged metric trace and spatial harmonic gauges}

To connect the unimodular Faddeev-Popov gauge (3.21) to averaged metric trace and spatial harmonic gauge (3.31) we derive the transformation functional parameter as follows

$$
\theta^{\prime}[\phi]=-\int d^{4} x\left[-\bar{c}^{*} \sqrt{h}\left(\overline{\ln h}-\bar{\Phi}-\overline{\operatorname{tr}\left(h_{i j}\right)}\right)+c_{i}^{*} \sqrt{h}\left(-\frac{1}{2} d^{i j k} h_{j k}-\partial_{j}\left(\sqrt{h} h^{i j}\right)\right)\right] .
$$


With this parameter the Jacobian of functional measure is calculated by

$$
\begin{aligned}
J[\phi]= & \exp \left[\int d ^ { 4 } x \left(\sqrt{h} \bar{\eta}(\overline{\ln h}-\bar{\Phi})+\frac{1}{2} \sqrt{h} \eta_{i} d^{i j k} h_{j k}+\sqrt{h} \bar{c}^{*}(\overline{\ln h}-\bar{\Phi}+2)\left(K \bar{c}+D_{i} c^{i}\right)\right.\right. \\
& +\sqrt{h} c_{i}^{*} d^{i j k}\left(K_{j k}+\frac{1}{2} h_{j k} K\right) \bar{c}+\frac{1}{2} \sqrt{h} c_{i}^{*} d^{i j k}\left(h_{j k} D_{l} c^{l}+\partial_{l} h_{j k} c^{l}+h_{j l} \partial_{k} c^{l}+h_{l k} \partial_{j} c^{l}\right) \\
& -\sqrt{h} \bar{\eta} \overline{\operatorname{tr}\left(h_{i j}\right)}-\eta_{i} \partial_{j}\left(\sqrt{h} h^{i j}\right)-\sqrt{h} \bar{c}^{*} \overline{\operatorname{tr}\left(h_{i j}\right)}\left(K \bar{c}+D_{i} c^{i}\right) \\
& -\sqrt{h} \bar{c}^{*} \sum_{i}\left(2 K_{i i} \bar{c}+\partial_{j} h_{i i} c^{j}+2 h_{i j} \partial_{i} c^{j}\right)+2 c_{i}^{*} \partial_{j}\left[\sqrt{h}\left(K^{i j}-\frac{1}{2} h^{i j} K\right) \bar{c}\right] \\
& -c_{i}^{*} \partial_{j} \partial_{k}\left(\sqrt{h} h^{i k}\right) c^{j}-c_{i}^{*} \partial_{j}\left(\sqrt{h} h^{i j}\right) \partial_{k} c^{k}+c_{i}^{*} \partial_{j}\left(\sqrt{h} h^{j k}\right) \partial_{k} c^{i} \\
& \left.\left.+c_{i}^{*} \sqrt{h} h^{j k} \partial_{j} \partial_{k} c^{i}\right)\right] .
\end{aligned}
$$

This Jacobian (4.30) amounts the following change into the expression of transition amplitude (3.27)

$$
\begin{aligned}
\mathcal{N}^{-1} \int \mathcal{D} \Phi^{\prime} e^{\frac{i}{\hbar}\left(S_{E H}\left[\phi^{\prime}\right]+S_{g f+g h}^{F P}\right)} & =\mathcal{N}^{-1} \int J[\phi] \mathcal{D} \Phi e^{\frac{i}{\hbar}\left(S_{E H}[\phi]+S_{g f+g h}^{F P}\right)} \\
& =\mathcal{N}^{-1} \int \mathcal{D} \Phi e^{\frac{i}{\hbar}\left(S_{E H}[\phi]+S_{g f+g h}^{T H}\right)} \\
& =Z_{\mathrm{UG}}^{T H} .
\end{aligned}
$$

This relation assures the connection (under FFBRST transformation) between path integrals in the unimodular Faddeev-Popov and averaged metric trace and spatial harmonic gauges, Eqs.(3.27) and (3.34), respectively.

\subsubsection{Averaged metric determinant to averaged metric trace gauge}

Finally, we establish a connection between averaged metric determinant to averaged metric trace gauges, Eqs. 3.28) and (3.31), respectively. For this purpose, we construct the following infinitesimal field-dependent parameter:

$$
\theta^{\prime}[\phi]=-\int d^{4} x\left[-\bar{c}^{*} \sqrt{h}\left(\overline{\ln h}-\bar{\Phi}-\overline{\operatorname{tr}\left(h_{i j}\right)}\right)\right] .
$$

The Jacobian expression 4.12 together with 4.32 yields

$$
\begin{aligned}
J[\phi]= & \exp \left[\int d ^ { 4 } x \left(\sqrt{h} \bar{\eta}(\overline{\ln h}-\bar{\Phi})+\sqrt{h} \bar{c}^{*}(\overline{\ln h}-\bar{\Phi}+2)\left(K \bar{c}+D_{i} c^{i}\right)\right.\right. \\
& -\sqrt{h} \bar{\eta} \overline{\operatorname{tr}\left(h_{i j}\right)}-\sqrt{h} \bar{c}^{*} \overline{\operatorname{tr}\left(h_{i j}\right)}\left(K \bar{c}+D_{i} c^{i}\right) \\
& \left.\left.-\sqrt{h} \bar{c}^{*} \sum_{i}\left(2 K_{i i} \bar{c}+\partial_{j} h_{i i} c^{j}+2 h_{i j} \partial_{i} c^{j}\right)\right)\right] .
\end{aligned}
$$


It can directly be seen that this Jacobian (4.33) is responsible for the connection of averaged metric determinant gauge to averaged metric trace gauge as follows

$$
\begin{aligned}
\mathcal{N}^{-1} \int \mathcal{D} \Phi^{\prime} e^{\frac{i}{\hbar}\left(S_{E H}\left[\phi^{\prime}\right]+S_{g f+g h}^{D H}\right)} & =\mathcal{N}^{-1} \int J[\phi] \mathcal{D} \Phi e^{\frac{i}{\hbar}\left(S_{E H}[\phi]+S_{g f+g h}^{D H}\right)} \\
& =\mathcal{N}^{-1} \int \mathcal{D} \Phi e^{\frac{i}{\hbar}\left(S_{E H}[\phi]+S_{g f+g h}^{T H}\right)} \\
& =Z_{\mathrm{UG}}^{T H} .
\end{aligned}
$$

Thus we conclude this subsection where we have explicitly presented a detailed analysis concerning the FFBRST transformation equivalence (with specific choices for the parameters) relating various gauges of the unimodular gravity with fixed metric determinant.

\section{Concluding Remarks}

As we know a gauge invariant theory can not be quantized correctly without fixing the gauge properly. Being a gauge theory, we have discussed the implementation of various gauge conditions for two version of the unimodular gravitational theory, fully diffeomorphism-invariant unimodular gravity and unimodular gravity with fixed metric determinant. We have further incorporated these gauges together with ghost terms at quantum level by defining the respective path integral. Further on, we derived the nilpotent BRST symmetry for the effective action as well as for the transition amplitude.

In particular, it should be noted that, in the fully diffeomorphism invariant unimodular gravity [11], after the auxiliary variables of action (2.4) have been integrated out, the gauge symmetry of the path integral 2.5) is the same as that of GR. Therefore the formulation of gauge conditions and the associated gauge fixing and ghost actions can be achieved in a familiar way. We obtained the gauge fixing and ghost action for several relevant gauges in section 2. The results can be applied to both (DUG) unimodular gravity and GR due to the similar gauge symmetry.

Furthermore, we have formulated three possible gauges for unimodular gravity theory with fixed metric determinant (3.1) in section 3. In this case, gauge fixing is more involving since the gauge symmetry of the theory has been restricted, so that the unimodular condition remains gauge invariant. Consequently, the integral of the Hamiltonian constraint over space is not a generator of a gauge transformation, and hence the integral of one of the gauge conditions must vanish, and the corresponding ghost and antighost fields are average-free as well (see [11 for a detailed analysis). In some cases, this restricted gauge structure may complicate the formulation of gauge conditions and BRST invariant actions, in particular, if the chosen gauge conditions involve the canonical momentum conjugate to the induced metric on the spatial hypersurface; an example of this problem is discussed in Appendix A.

The BRST symmetry of these theories has been further extended by making the transformation parameter finite and field-dependent. We have shown that the FFBRST transformation of the Jacobian of the invariant functional measure, with specific choices for the transformation parameter, connects various gauges of both given unimodular theories of gravity. This establishes a way to consistently relate several path integral expressions when defined in different gauge conditions. However, we should emphasize that we are using the FFBRST transformation only for connecting different welldefined gauges, since then there should be no physical change in the quantum theory. Thus FFBRST 
formulation discussed here could be useful in comparing results in two gauges for unimodular gravity theories.

\section{Acknowledgments}

M.O. thankfully acknowledges support from the Emil Aaltonen Foundation. R.B. acknowledges FAPESP for full support, Project No. 2011/20653-3.

\section{A Unimodular Dirac gauge}

In order to justify the absence of the Dirac gauge in our analysis of unimodular gravity with fixed metric determinant, we highlight a problem in the formulation of a gauge condition that depends on the canonical momentum $\pi^{i j}$ conjugate to the induced metric $h_{i j}$. The Dirac gauge could be defined in the unimodular setting as

$$
\bar{\chi}_{\mathrm{D}}^{0}=\overline{h_{i j} \pi^{i j}}=h_{i j} \pi^{i j}-\frac{\sqrt{h}}{\int_{\Sigma_{t}} \sqrt{h}} \int_{\Sigma_{t}} h_{i j} \pi^{i j} \approx 0, \quad \chi_{\mathrm{D}}^{i}=\partial_{j}\left(h^{\frac{1}{3}} h^{i j}\right) \approx 0 .
$$

The BRST invariant gauge and ghost action for these gauge conditions can be written in the form

$$
S_{g f+g h}^{U D}=\int d^{4} x\left(-\bar{\eta} \bar{\chi}_{\mathrm{D}}^{0}-\eta_{i} \chi_{\mathrm{D}}^{i}-\bar{c}^{*} s_{b} \bar{\chi}_{\mathrm{D}}^{0}-c_{i}^{*} s_{b} \chi_{\mathrm{D}}^{i}\right)
$$

where the pair of ghosts $\bar{c}, \bar{c}^{*}$ are average-free, while the ghosts $c^{i}, c_{j}^{*}$ are not.

Let us start by computing the Slanov variation of the gauge conditions $\chi_{\mathrm{D}}^{i}$. This demand some direct calculation that results into

$$
\begin{aligned}
s_{b} \chi_{\mathrm{D}}^{i}= & -2 \kappa \partial_{j}\left[h^{-\frac{1}{6}}\left(\pi^{i j}-\frac{1}{3} h^{i j} h_{k l} \pi^{k l}\right) \bar{c}\right]+\frac{2}{3} \chi_{\mathrm{D}}^{i} D_{j} c^{j}-\chi_{\mathrm{D}}^{j} D_{j} c^{i} \\
& -h^{\frac{1}{3}}\left(\delta_{j}^{i} h^{k l} \partial_{k} \partial_{l}+\frac{1}{3} h^{i k} \partial_{k} \partial_{j}\right) c^{j} .
\end{aligned}
$$

Next we proceed to compute the Slanov variation of the gauge condition $\bar{\chi}_{\mathrm{D}}^{0}$,

$$
s_{b} \bar{\chi}_{\mathrm{D}}^{0}=s_{b}\left(h_{i j} \pi^{i j}\right)-s_{b} \sqrt{h}\left(\frac{1}{\int_{\Sigma_{t}} \sqrt{h}} \int_{\Sigma_{t}} h_{i j} \pi^{i j}\right)-\sqrt{h} s_{b}\left(\frac{1}{\int_{\Sigma_{t}} \sqrt{h}} \int_{\Sigma_{t}} h_{i j} \pi^{i j}\right),
$$

where the last term of the above expression drops out of the action A.2, since the ghost $\bar{c}^{*}$ has a vanishing average.

After evaluating the respective variation, we can use the resulting expression (A.4) in order to write the third term of the action A.2 in the following form

$$
\begin{aligned}
\int d^{4} x \bar{c}^{*} s_{b} \bar{\chi}_{\mathrm{D}}^{0}= & \int d^{4} x \bar{c}^{*}\left(\frac{3}{2} \overline{\mathcal{H}}_{T} \bar{c}-\frac{2}{\kappa} \sqrt{h}\left(D^{i} D_{i}-{ }^{(3)} R\right) \bar{c}+\partial_{k}\left(h_{i j} \pi^{i j} c^{k}\right)\right) \\
& +3 \int d^{4} x \sqrt{h} \bar{c}^{*} \bar{c}\left(\frac{1}{\int_{\Sigma_{t}} \sqrt{h}} \int_{\Sigma_{t}}\left[\frac{\kappa}{\sqrt{h}} \pi^{i j} \mathcal{G}_{i j k l} \pi^{k l}-\frac{\sqrt{h}}{\kappa}(3) R\right]\right) \\
& -\int d^{4} x \bar{c}^{*}\left(-\frac{\kappa}{2} h_{i j} \pi^{i j} \bar{c}+\sqrt{h} D_{i} c^{i}\right)\left[\frac{1}{\int_{\Sigma_{t}} \sqrt{h}} \int_{\Sigma_{t}} h_{i j} \pi^{i j}\right]
\end{aligned}
$$


This is a problematic result, since it contains quadratic terms in $\pi^{i j}$ that are not constraints. In the path integral, the Faddeev-Popov determinant should be at most linear in $\pi^{i j}$ so that the (gaussian) integration over the momenta can be performed. The quadratic terms should involve a constraint so that they can be absorbed via shifts of Lagrange multipliers. Above only the constraint term $\frac{3}{2} \overline{\mathcal{H}}_{T}$ appears, while the integrated term is not a constraint. Indeed we could use the constraint $\mathcal{H}_{0}$ to write

$$
\frac{1}{\int_{\Sigma_{t}} \sqrt{h}} \int_{\Sigma_{t}}\left(\frac{\kappa}{\sqrt{h}} \pi^{i j} \mathcal{G}_{i j k l} \pi^{k l}-\frac{\sqrt{h}}{\kappa}{ }^{(3)} R\right)=\frac{\mathcal{H}_{0}}{\int_{\Sigma_{t}} \sqrt{h}}-\lambda_{0},
$$

but then the cosmological constant variable $\lambda_{0}$ reappears, which is not correct since it is integrated

in the path integral to obtain the averaged unimodular condition factor $\delta\left(\int_{\Sigma_{t}}\left(\sqrt{-g}-\epsilon_{0}\right)\right)$ 11]. The last term in A.5 is equally problematic, since it also involves a quadratic $\pi^{i j}$ term, which is not a constraint.

\section{References}

[1] S. Weinberg, "The Cosmological Constant Problem," Rev. Mod. Phys. 61, 1 (1989).

[2] T. Padmanabhan, "Cosmological constant: The Weight of the vacuum," Phys. Rept. 380, 235 (2003), arXiv:hep-th/0212290.

[3] R. Bousso, "TASI Lectures on the Cosmological Constant," Gen. Rel. Grav. 40, 607 (2008), arXiv:0708.4231 [hep-th].

[4] A. Einstein, "The Foundation of the General Theory of Relativity," Annalen Phys. 49, 769 (1916), translated and included in The Principle of Relativity, by H.A. Lorentz et al. (Dover Press, New York, 1923).

[5] A. Einstein, "Do gravitational fields play an essential part in the structure of the elementary particles of matter?," Sitzungsber. Preuss. Akad. Wiss. Berlin (Math. Phys.) 1919, 433 (1919), translated and included in The Principle of Relativity, by H.A. Lorentz et al. (Dover Press, New York, 1923).

[6] L. Smolin, "Quantization of unimodular gravity and the cosmological constant problems," Phys. Rev. D 80, 084003 (2009), arXiv:0904.4841 [hep-th].

[7] W. Buchmuller and N. Dragon, "Einstein gravity from restricted coordinate invariance," Phys. Lett. B 207, 292 (1988),

[8] W. G. Unruh, "A Unimodular Theory of Canonical Quantum Gravity," Phys. Rev. D 40, 1048 $(1989)$.

[9] K. V. Kuchar, "Does an unspecified cosmological constant solve the problem of time in quantum gravity?," Phys. Rev. D 43, 3332 (1991).

[10] M. Henneaux and C. Teitelboim, "The Cosmological Constant and General Covariance," Phys. Lett. B 222, 195 (1989). 
[11] R. Bufalo, M. Oksanen and A. Tureaun, "How unimodular gravity theories differ from general relativity at quantum level," Eur. Phys. J. C 75 (2015) 10, 477, arXiv:1505.04978 [hep-th],

[12] Y. J. Ng and H. van Dam, "Possible solution to the cosmological constant problem," Phys. Rev. Lett. 65, 1972 (1990).

[13] R. D. Sorkin, "On the Role of Time in the Sum Over Histories Framework for Gravity," Int. J. Theor. Phys. 33, 523 (1994), Originally presented at the conference, The History of Modern Gauge Theories, held at Logan, Utah, July 1987.

[14] W. G. Unruh and R. M. Wald, "Time and the Interpretation of Canonical Quantum Gravity," Phys. Rev. D 40, 2598 (1989).

[15] A. Álvarez, S. González-Martín, M. Herrero-Valea and C. P. Martín, "Quantum Corrections to Unimodular Gravity," JHEP 1508, 078 (2015), arXiv:1505.01995 [hep-th].

[16] S. D. Joglekar and B. P. Mandal, "Finite field dependent BRS transformations," Phys. Rev. D 51, 1919 (1995).

[17] S. D. Joglekar and A. Misra, "Correct treatment of $1 /(\eta \cdot k)^{p}$ singularities in the axial gauge propagator," Int. J. Mod. Phys. A 15, 1453 (2000), arXiv:hep-th/9909123.

[18] S. D. Joglekar and B. P. Mandal, "Application of finite field dependent BRS transformations to problems of the Coulomb gauge," Int. J. Mod. Phys. A 17, 1279 (2002), arXiv:hep-th/0105042.

[19] S. Upadhyay, S. K. Rai and B. P. Mandal, "Off-shell nilpotent finite BRST/anti-BRST transformations," J. Math. Phys. 52, 022301 (2011), arXiv:1002.1373 [hep-th],

[20] V. N. Gribov, "Quantization of non-Abelian gauge theories," Nucl. Phys. B 139, 1 (1978),

[21] D. Zwanziger, "Local and renormalizable action from the gribov horizon," Nucl. Phys. B 323, $513(1989)$.

[22] D. Zwanziger, "Renormalizability of the critical limit of lattice gauge theory by BRS invariance," Nucl. Phys. B 399, 477 (1993).

[23] S. Upadhyay and B. P. Mandal, "Generalized BRST symmetry for arbitrary spin conformal field theory," Phys. Lett. B 744, 231 (2015), arXiv:1409.1735 [hep-th];

S. Upadhyay and B. P. Mandal, "Gaugeon formalism in the framework of generalized BRST symmetry," Prog. Theor. Exp. Phys. 053B04, 1 (2014), arXiv:1403.6194 [hep-th];

S. Upadhyay and B. P. Mandal, "Field dependent nilpotent symmetry for gauge theories," Eur. Phys. J. C 72, 2065 (2012), arXiv:1201.0084 [hep-th];

S. Upadhyay and B. P. Mandal, "Finite BRST transformation and constrained systems," Annls. Phys. 327, 2885 (2012), arXiv:1207.6449 [hep-th];

S. Upadhyay and B. P. Mandal, "Relating Gribov-Zwanziger theory to effective Yang-Mills theory," Eur. Phys. Lett. 93, 31001 (2011), arXiv:1101.5448 [hep-th];

S. Upadhyay and B. P. Mandal, "Generalized BRST transformation in Abelian rank-2 antisymmetric tensor field theory," Mod. Phys. Lett. A 25, 3347 (2010), arXiv:1004.0330 [hep-th], 
[24] S. Upadhyay, M. K. Dwivedi and B. P. Mandal, "The noncovariant gauges in 3-form theories," Int. J. Mod. Phys. A 28, 1350033 (2013), arXiv:1301.0222 [hep-th],

[25] S. Upadhyay, M. K. Dwivedi and B. P. Mandal, "Emergence of Lowenstein-Zimmermann mass terms for $\mathrm{QED}_{3}, "$ arXiv:1407.2017 [hep-th],

[26] M. Faizal, B. P. Mandal and S. Upadhyay, "Finite BRST Transformations for the BaggerLambert-Gustavasson Theory," Phys. Lett. B 721, 159 (2013), arXiv:1212.5653 [hep-th].

[27] S. Upadhyay and D. Das, "ABJM theory in Batalin-Vilkovisky formulation," Phys. Lett. B 733, 63 (2014), arXiv:1404.2633 [hep-th].

[28] M. Faizal, S. Upadhyay and B. P. Mandal, "Finite field-dependent BRST symmetry for ABJM theory in $\mathcal{N}=1$ superspace," Phys. Lett. B 738, 201 (2014), arXiv:1410.0671 [hep-th];

M. Faizal, S. Upadhyay and B. P. Mandal, "IR finite graviton propagators in de Sitter spacetime," Eur. Phys. J. C 76, 189 (2016), arXiv:1604.00390 [hep-th],

M. Faizal, S. Upadhyay and B. P. Mandal, "Anti-FFBRST Transformations for the BLG Theory in Presence of a Boundary," Int. J. Mod. Phys. A 30, 1550032 (2015), arXiv:1501.01616 [hep-th],

[29] J. F. Bagger and N. Lambert, "Modeling multiple M2-branes," Phys. Rev. D 75, 045020 (2007), arXiv:hep-th/0611108;

J. F. Bagger and N. Lambert, "Gauge symmetry and supersymmetry of multiple M2-branes ," Phys. Rev. D 77, 065008 (2008), arXiv:0711.0955 [hep-th];

J. F. Bagger and N. Lambert, "Comments on multiple M2-branes ," JHEP 0802, 105 (2008), arXiv:0712.3738 [hep-th].

[30] A. Gustavsson, "Algebraic structures on parallel M2 branes," Nucl. Phys. B 811, 66 (2009), arXiv:0709.1260 [hep-th].

[31] O. Aharony, O. Bergman, D. L. Jafferis and J. Maldacena, "N=6 superconformal Chern-Simonsmatter theories, M2-branes and their gravity duals," JHEP 0810, 091 (2008), arXiv:0806.1218 [hep-th].

[32] R. Banerjee and S. Upadhyay, "Generalized supersymmetry and sigma models," Phys. Lett. B 734, 369 (2014), arXiv:1310.1168 [hep-th],

[33] B. P. Mandal, S. K. Rai and S. Upadhyay, "Finite nilpotent symmetry in Batalin-Vilkovisky formalism," Eur. Phys. Lett. 92, 21001 (2010), arXiv:1009.5859 [hep-th]

S. Upadhyay, "Super-group field cosmology in Batalin-Vilkovisky formulation," Int J Theor Phys (2016), arXiv:1606.09606 [hep-th];

S. Upadhyay and P. A. Ganai, "Finite Field-dependent Symmetry in Thirring Model," Prog. Theor. Exp. Phys. 063B04, 1 (2016), arXiv:1605.04290 [hep-th],

[34] S. Upadhyay, "The conformal gauge to the derivative gauge for worldsheet gravity," Phys. Lett. B 740, 341 (2015), arXiv:1412.5911 [hep-th];

S. Upadhyay, "Field-dependent symmetries in Friedmann-Robertson-Walker models," Annals Phys. 356, 299 (2015), arXiv:1503.04197 [hep-th]; 
S. Upadhyay, "Nilpotent Symmetries in Super-Group Field Cosmology," Mod. Phys. Lett. A 30, 1550072 (2015), arXiv:1502.05217 [hep-th];

S. Upadhyay, "Finite field-dependent symmetries in perturbative quantum gravity," Annals Phys. 340, 110 (2014), arXiv:1310.8579 [hep-th];

S. Upadhyay, "Generalized BRST Symmetry and Gaugeon Formalism for Perturbative Quantum Gravity: Novel Observation," Annals Phys. 344, 290 (2014), arXiv:1403.6166 [hep-th];

S. Upadhyay, "Field-dependent quantum gauge transformation," Eur. Phys. Lett. 105, 21001 (2014), arXiv:1402.3373 [hep-th];

S. Upadhyay, "N=1 super-Chern-Simons theory in Batalin-Vilkovisky formulation," Eur. Phys. Lett. 104, 61001 (2013), arXiv:1401.1968 [hep-th];

S. Upadhyay, "Aspects of finite field-dependent symmetry in SU(2) Cho-Faddeev-Niemi decomposition," Phys. Lett. B 727, 293 (2013), arXiv:1310.2013 [hep-th].

[35] M. Henneaux, C. Teitelboim, Quantization of gauge systems, Princeton, USA: Univ. Press (1992).

[36] P. M. Lavrov and O. Lechtenfeld, "Field-dependent BRST transformations in Yang-Mills theory," Phys. Lett. B 725, 382 (2013), arXiv:1305.0712 [hep-th],

[37] P. Y. Moshin and A. A. Reshetnyak, "Field-dependent BRST-antiBRST transformations in generalized Hamiltonian formalism," Int. J. Mod. Phys. A 29, 1450159 (2014), arXiv:1405.7549 [hep-th],

[38] P. Y. Moshin and A. A. Reshetnyak, "Field-dependent BRSTanti-BRST Lagrangian transformations," Int. J. Mod. Phys. A 30, 1550021 (2015), arXiv:1406.5086 [hep-th].

[39] P. Y. Moshin and A. A. Reshetnyak, "Finite BRST-antiBRST Transformations in Lagrangian Formalism," Phys. Lett. B 739, 110 (2014), arXiv:1406.0179 [hep-th].

[40] S. Upadhyay, A. Reshetnyak and B. P. Mandal, "Comments on interactions in the SUSY models," Eur. Phys. J. C 76, 391 (2016), arXiv:1605.02973v5 [physics.gen-ph],

[41] P. Y. Moshin and A. A. Reshetnyak, "Field-dependent BRST-antiBRST Transformations in YangMills and Gribov-Zwanziger Theories," Nucl. Phys. B 888, 92 (2014), arXiv:1405.0790 [hep-th].

[42] P. Y. Moshin and A. A. Reshetnyak, "Finite Field-Dependent BRST-antiBRST Transformations: Jacobians and Application to the Standard Model," arXiv:1506.04660 [hep-th],

[43] K. Nishijima and M. Okawa, "The Becchi-Rouet-Stora Transformation for the Gravitational Field," Prog. Theor. Phys. 60, 272 (1978).

[44] L. D. Faddeev and V. N. Popov, "Covariant quantization of the gravitational field," Sov. Phys. Usp. 74, 777 (1974). 